\title{
On surgery along Brunnian links in 3-manifolds
}

\author{
Jean-Baptiste Meilhan
}

\begin{abstract}
We consider surgery moves along $(n+1)$-component Brunnian links in compact connected oriented 3-manifolds, where the framing of the components is in $\left\{\frac{1}{k} ; k \in\right.$ $\mathbf{Z}\}$. We show that no finite type invariant of degree $<2 n-2$ can detect such a surgery move. The case of two link-homotopic Brunnian links is also considered. We relate finite type invariants of integral homology spheres obtained by such operations to Goussarov-Vassiliev invariants of Brunnian links.
\end{abstract}

$57 \mathrm{~N} 10 ; 57 \mathrm{M} 27$

\section{Introduction}

In [19], Ohtsuki introduced the notion of finite type invariants of integral homology spheres as an attempt to unify the topological invariants of these objects, in the same way as Goussarov-Vassiliev invariants provide a unified point of view on invariants of knots and links. This theory was later generalized to all oriented 3-manifolds by Cochran and Melvin [2].

Goussarov and Habiro developed independently another finite type invariants theory for compact connected oriented 3-manifolds, which essentially coincides with the Ohtsuki theory in the case of integral homology spheres $[4 ; 7 ; 12]$. This theory comes equipped with a new and powerful tool called calculus of clasper, which uses embedded graphs carrying some surgery instruction. Surgery moves along claspers define a family of (finer and finer) equivalence relations among 3-manifolds, called $Y_{k}$-equivalence, which gives a good idea of the information contained by finite type invariants: two compact connected oriented 3-manifolds are not distinguished by invariants of degree $<k$ if they are $Y_{k}$-equivalent $[8 ; 12]$. These two conditions become equivalent when dealing with integral homology spheres.

Recall that a link $L$ is Brunnian if any proper sublink of $L$ is trivial. In some sense, an $n$-component Brunnian link is a 'pure $n$-component linking'. In this paper we consider those compact connected oriented 3-manifolds which are obtained by surgery along a Brunnian link. For a fixed number of components, we study which finite type invariants (ie of which degree) can vary under such an operation. 
Let $m=\left(m_{1}, \ldots, m_{n}\right) \in \mathbf{Z}^{n}$ be a collection of $n$ integers. Given a null-homologous, ordered $n$-component link $L$ in a compact connected oriented 3-manifold $M$, denote by $(L, m)$ the link $L$ with framing $\frac{1}{m_{i}}$ on the $i^{t h}$ component $; 1 \leq i \leq n$. We denote by $M_{(L, m)}$ the 3-manifold obtained from $M$ by surgery along the framed link $(L, m)$. We say that $M_{(L, m)}$ is obtained from $M$ by $\frac{1}{m}$-surgery along the link $L$.

Theorem 1.1 Let $n \geq 2$ and $m \in \mathbf{Z}^{n+1}$. Let $L$ be an $(n+1)$-component Brunnian link in a compact, connected, oriented 3-manifold $M$.

For $n=2, M_{(L, m)}$ and $M$ are $Y_{1}$-equivalent.

For $n \geq 3, M_{(L, m)}$ and $M$ are $Y_{2 n-2}$-equivalent. Consequently, they cannot be distinguished by any finite type invariant of degree $<2 n-2$.

Note that, for any Brunnian link $L$ in $M$, we have $M_{(L, m)} \cong M$ if $m_{i}=0$ for some $1 \leq i \leq n+1$. In this case, the statement is thus vacuous.

Two links are link-homotopic if they are related by a sequence of isotopies and selfcrossing changes, ie, crossing changes involving two strands of the same component. We obtain the following.

Theorem 1.2 Let $n \geq 2$ and $m \in \mathbf{Z}^{n+1}$. Let $L$ and $L^{\prime}$ be two link-homotopic $(n+1)$-component Brunnian links in a compact, connected, oriented 3-manifold $M$. Then $M_{(L, m)}$ and $M_{\left(L^{\prime}, m\right)}$ are $Y_{2 n-1}$-equivalent. Consequently, they cannot be distinguished by any finite type invariant of degree $<2 n-1$.

Actually, for integral homology spheres, the theorem is still true when " $2 n-1$ " is replaced by " $2 n$ ". (It follows from the last observation of Section 3.7.)

In the latter part of the paper, we study the relation between the above results and Goussarov-Vassiliev invariants of Brunnian links.

Let $\mathbf{Z} \mathcal{L}(n)$ be the free $\mathbf{Z}$-module generated by the set of isotopy classes of $n-$ component links in $S^{3}$. The theory of Goussarov-Vassiliev invariants of links involves a descending filtration

$$
\mathbf{Z} \mathcal{L}(n)=J_{0}(n) \supset J_{1}(n) \supset J_{2}(n) \supset \ldots
$$

called Goussarov-Vassiliev filtration (see Section 5.2). In a previous paper, Habiro and the author introduced the so-called Brunnian part $\operatorname{Br}\left(\bar{J}_{2 n}(n+1)\right)$ of $J_{2 n}(n+$ 1) $/ J_{2 n+1}(n+1)$, which is defined as the $\mathbf{Z}$-submodule generated by elements $[L-$ 
$U]_{J_{2 n+1}}$ where $L$ is an $(n+1)$-component Brunnian link and $U$ is the $(n+1)-$ component unlink [14]. Further, we constructed a linear map

$$
h_{n}: \mathcal{A}_{n-1}^{c}(\varnothing) \longrightarrow \operatorname{Br}\left(\bar{J}_{2 n}(n+1)\right),
$$

where $\mathcal{A}_{n-1}^{c}(\varnothing)$ is a $\mathbf{Z}$-module of connected trivalent diagrams with $2 n-2$ vertices. $h_{n}$ is an isomorphism over $\mathbf{Q}$ for $n \geq 2$. See Section 5 for precise definitions.

Let $\overline{\mathcal{S}}_{k}$ be the abelian group of $Y_{k+1}$-equivalence classes of integral homology spheres which are $Y_{k}$-equivalent to $S^{3} \cdot \overline{\mathcal{S}}_{2 k+1}=0$ for all $k \geq 1$, and it is well known that $\overline{\mathcal{S}}_{2 k}$ is isomorphic to $\mathcal{A}_{k}^{c}(\varnothing)$ when tensoring by $\mathbf{Q}$. See Section 6.3. There is therefore an isomorphism over $\mathbf{Q}$ from $\operatorname{Br}\left(\bar{J}_{2 n}(n+1)\right)$ to $\overline{\mathcal{S}}_{2 n-2}$, for $n \geq 2$. The next theorem states that this isomorphism is induced by $(+1)$-framed surgery.

For a null-homologous ordered link $L$ in a compact connected oriented 3-manifold $M$, denote by $(L,+1)$ the link $L$ with all components having framing +1 .

Theorem 1.3 For $n \geq 2$, the assignment

$$
[L-U]_{J_{2 n+1}} \mapsto\left[S_{(L,+1)}^{3}\right]_{Y_{2 n-1}}
$$

defines an isomorphism

$$
\kappa_{n}: \operatorname{Br}\left(\bar{J}_{2 n}(n+1)\right) \otimes \mathbf{Q} \longrightarrow \overline{\mathcal{S}}_{2 n-2} \otimes \mathbf{Q} .
$$

We actually show that these two $\mathbf{Q}$-modules are isomorphic to the so-called 'connected part' of the Ohtsuki filtration, by using the abelian group $\mathcal{A}_{n-1}^{c}(\varnothing)$. See Section 6 for definitions and statements.

The rest of this paper is organized as follows.

In Section 2, we give a brief review of the theory of claspers, both for compact connected oriented 3-manifolds and for links in a fixed manifold. In Section 3, we study the $Y_{k}$-equivalence class of integral homology spheres obtained by surgery along claspers with several special leaves. This section can be read separately from the rest of the paper and might be of independent interest. In Section 4, we use the main result of section 3 to prove Theorems 1.1 and 1.2. In Section 5, we recall several results obtained by Habiro and the author in [14]. In Section 6, we define the material announced above and prove Theorem 1.3. In Section 7, we give the (technical) proof of Proposition 3.8.

Acknowledgments The author is grateful to Kazuo Habiro for many helpful conversations and comments on an early version of this paper. He was supported by a Postdoctoral Fellowship and a Grant-in-Aid for Scientific Research of the Japan Society for the Promotion of Science.

Algebraic $8 \mathcal{G}$ Geometric Topology, Volume 6 (2006) 


\section{Claspers}

Throughout this paper, all 3-manifolds will be supposed to be compact, connected and oriented.

\subsection{Clasper theory for 3-manifolds}

Let us briefly recall from $[4 ; 7 ; 12]$ the fundamental notions of clasper theory for 3-manifolds.

Definition 2.1 A clasper in a 3-manifold $M$ is an embedding

$$
G: F \longrightarrow \text { int } M
$$

of a compact (possibly unorientable) surface $F$. $F$ is decomposed into constituents connected by disjoint bands called edges. Constituents are disjoint connected subsurfaces, either annuli or disks:

- A leaf is an annulus with one edge attached.

- A node is a disk with three edges attached.

- A box is a disk with at least three edges attached, one being distinguished with the others. This distinction is done by drawing a box as a rectangle.

Observe that this definition slightly extends the one in [12], where a box has always three edges attached.

We will make use of the drawing convention for claspers of [12, Figure 7], except for the following: $\mathrm{a} \oplus$ (resp. $\ominus$ ) on an edge represents a positive (resp. negative) half-twist. This replaces the convention of a circled $S$ (resp. $S^{-1}$ ) used in [12].

2.1.1 Surgery along claspers Given a clasper $G$ in $M$, we can construct, in a regular neighborhood of the clasper, an associated framed link $L_{G}$ as follows. First, replace each node and box of $G$ by leaves as shown in Figure 2.1 (a) and (b). We obtain a union of I-shaped claspers, one for each edge of $G . L_{G}$ is obtained by replacing each of these I-shaped claspers by a 2-component framed link as shown in Figure 2.1 (c). ${ }^{1}$

Surgery along the clasper $G$ is defined to be surgery along $L_{G}$.

In [12, Proposition 2.7], Habiro gives a list of 12 moves on claspers which gives equivalent claspers, that is claspers with diffeomorphic surgery effect. We will freely use Habiro's moves (which are essentially derived from Kirby calculus) by referring to their numbering in Habiro's paper.

\footnotetext{
${ }^{1}$ Here and throughout the paper, blackboard framing convention is used.
} 


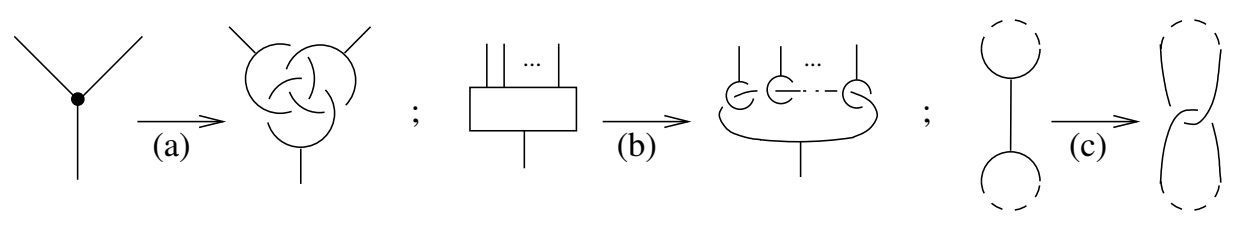

Figure 2.1: Constructing the framed link associated to a clasper

2.1.2 The $Y_{k}$-equivalence For $n \geq 1$, a $Y_{n}$-graph is a connected clasper $G$ without boxes and with $n$ nodes, where a connected clasper is a clasper whose underlying surface is connected. The integer $n$ is called the degree of $G$.

A $Y_{k}$-tree is a $Y_{k}$-graph $T$ such that the union of edges and nodes of $T$ is simply connected. For $k \geq 3$, we say that a $Y_{k}$-tree $T$ in a 3-manifold $M$ is linear if there is a 3-ball in $M$ which intersects the edges and nodes of $T$ as shown in Figure 2.2. The leaves denoted by $f$ and $f^{\prime}$ in the figure are called the ends of $T$.

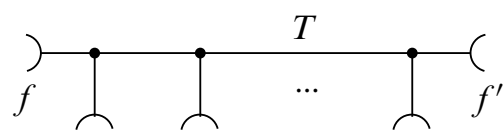

Figure 2.2: A linear tree $T$ and its two ends $f$ and $f^{\prime}$

A $Y_{k}$-forest is a clasper $T=T_{1} \cup \ldots \cup T_{p}(p \geq 0)$, where $T_{i}$ is a $Y_{k_{i}}-$ tree $(p \geq i \geq 1)$, such that $\min _{1 \leq i \leq p} k_{i}=k$.

A $Y_{k}$-subtree $T$ of a clasper $G$ is a connected union of leaves, nodes and edges of $G$ such that the union of edges and nodes of $T$ is simply connected and such that $T$ intersects $\overline{G \backslash T}$ along the attaching region of some edges of $T$, called branches.

A surgery move on $M$ along a $Y_{k}$-graph $G$ is called a $Y_{k}$-move. For example, a $Y_{1}$-move is equivalent to Matveev's Borromean surgery [16].

The $Y_{k}$-equivalence is the equivalence relation on 3-manifolds generated by $Y_{k}-$ moves and orientation-preserving diffeomorphisms. This equivalence relation becomes finer as $k$ increases: if $k \leq l$ and if $M \sim_{Y_{l}} N$, then we also have $M \sim_{Y_{k}} N$.

Recall that 'trees do suffice to define the $Y_{k}$-equivalence'. That is, $M \sim_{Y_{k}} N$ implies that there exists a $Y_{k}$-forest $F$ in $M$ such that $M_{F} \cong N$. 


\subsection{Clasper theory for links}

Another aspect of the theory of claspers is that it allows to study links in a fixed manifold. For this we use a slightly different type of claspers.

Definition 2.2 Let $L$ be a link in a 3-manifold $M$, and let $G$ be a clasper in $M$ which is disjoint from $L$. A disk-leaf of $G$ is a leaf $l$ of $G$ which is an unknot bounding a disk $D$ in $M$ with respect to which it is 0 -framed. ${ }^{2}$ We call $D$ the bounding disk of $f$. The interior of $D$ is disjoint from $G$ and from any other bounding disk, but it may intersect $L$ transversely. For convenience, we say that a disk-leaf $f$ intersects the link $L$ when its bounding disk does.

A $C_{n}$-tree (resp. linear $C_{n}$-tree) for a link $L$ in a 3 -manifold $M$ is a $Y_{n-1}$-tree (resp. linear $Y_{n-1}-$ tree) in $M$ such that each of its leaves is a disk-leaf.

Given a $C_{n}$-tree $C$ in $M$, there exists a canonical diffeomorphism between $M$ and the manifold $M_{C}$. So surgery along a $C_{n}$-tree can be regarded as a local move on links in the manifold $M$.

A $C_{n}$-tree $G$ for a link $L$ is simple (with respect to $L$ ) if each disk-leaf of $G$ intersects $L$ exactly once.

A surgery move on a link $L$ along a $C_{k}$-tree is called a $C_{k}$-move. The $C_{k}$-equivalence is the equivalence relation on links generated by the $C_{k}$-moves and isotopies. As in the case of manifolds, the $C_{n}$-equivalence relation implies the $C_{k}$-equivalence if $1 \leq k \leq n$. For more details, see [8;12].

\subsection{Some technical lemmas}

In this subsection, we state several technical lemmas about claspers.

First, we introduce several moves on claspers which produce equivalent claspers, like the 12 Habiro's moves. In each of the next three statements, the figure represents two claspers in a given 3-manifold which are identical outside a 3-ball, where they are as depicted.

Lemma 2.3 The move of Figure 2.3 produces equivalent claspers.

This is an immediate consequence of [4, Theorem 3.1] (taking into account that the convention used in [4] for the definition of the surgery link associated to a clasper is the opposite of the one used in the present paper).

Algebraic 83 Geometric Topology, Volume 6 (2006) 


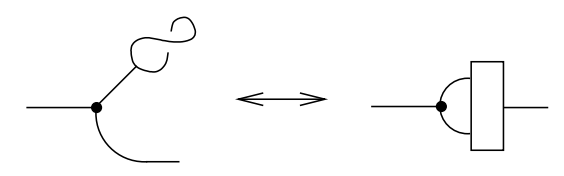

Figure 2.3

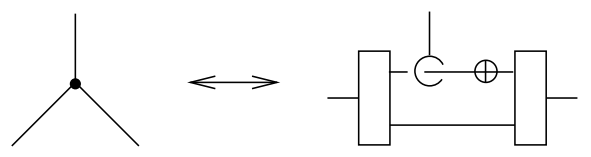

Figure 2.4

\section{Lemma 2.4 The move of Figure 2.4 produces equivalent claspers.}

This move is, in some sense, the inverse of Habiro's move 12. See also Figure 25 of [3], where a similar move appears.

Proof Consider the clasper on the right-hand side of Figure 2.4. By replacing the two boxes by leaves as shown in Figure 2.1 (b) and applying Habiro's move 1, we obtain the clasper depicted on the left-hand side of Figure 2.5. Now, the three leaves depicted

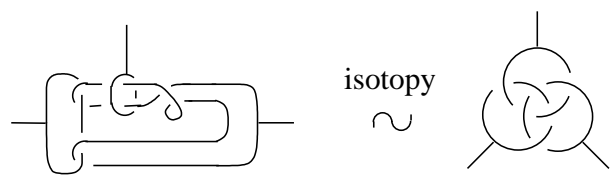

Figure 2.5

in this figure form a 3-component link which is isotopic to the Borromean link. As shown in Figure 2.1 (a), this is equivalent to a node.

Lemma 2.5 The moves of Figure 2.6 produce equivalent claspers.

This 'associativity' property of boxes is easily checked using Figure 2.1 (b) - see Figure 37 of [12].

\footnotetext{
${ }^{2}$ Here we regard a leaf, which is an embedded annulus, as a knot with a framing.
} 


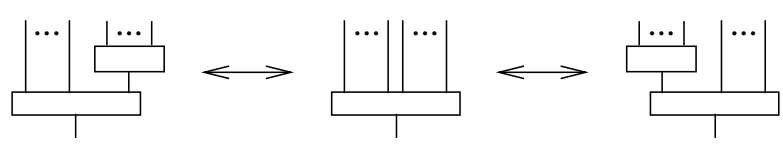

Figure 2.6: The associativity of boxes

The next lemma deals with crossing change operations on claspers. A crossing change is a local move as illustrated in Figure 2.7. The proof is omitted, as it uses the same techniques as in [12, Section 4] (where similar statements appear). See also [17, Section $1.4]$.

Lemma 2.6 Let $T_{1} \cup T_{2}$ be a disjoint union of a $Y_{k_{1}}$-tree and a $Y_{k_{2}}$-tree in a 3manifold $M$. Let $T_{1}^{\prime} \cup T_{2}^{\prime}$ be obtained by a crossing change $c$ of an edge or a leaf

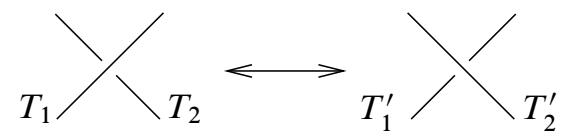

Figure 2.7: A crossing change

of $T_{1}$ with an edge or a leaf of $T_{2}$ (see Figure 2.7), and let $C \in\{0,1,2\}$ denotes the number of edges involved in the crossing change $c$. Then

(1) $M_{T_{1} \cup T_{2}} \sim Y_{k_{1}+k_{2}+C} M_{T_{1}^{\prime} \cup T_{2}^{\prime}}$.

(2) $M_{T_{1} \cup T_{2}} \sim Y_{k_{1}+k_{2}+C+1} M_{T_{1}^{\prime} \cup T_{2}^{\prime} \cup T}$, where $T$ is a parallel copy, disjoint from $T_{1}^{\prime} \cup T_{2}^{\prime}$, of some $Y_{k_{1}+k_{2}+C}$-tree $\tilde{T}$ obtained as follows:

(a) If $c$ involves an edge $e_{1}$ of $T_{1}$ and an edge $e_{2}$ of $T_{2}$, then $C=2$ and $\tilde{T}$ is obtained by inserting a node $n_{1}$ in $e_{1}$ and a node $n_{2}$ in $e_{2}$, and connecting $n_{1}$ and $n_{2}$ by an edge.

(b) If $c$ involves an edge $e$ of $T_{1}$ and a leaf $f$ of $T_{2}$, then $C=1$ and $\tilde{T}$ is obtained by inserting a node $n$ in $e$, and connecting $n_{1}$ to the edge incident to $f$.

(c) If $c$ involves a leaf $f_{1}$ of $T_{1}$ and a leaf $f_{2}$ of $T_{2}$, then $C=0$ and $\widetilde{T}$ is obtained by connecting the edges incident to $f_{1}$ and $f_{2}$.

Remark 2.7 This lemma is only valid for trees. However, if we are given graphs or subtrees instead, observe that it suffices to use Habiro's move 2 to obtain equivalent trees. So in this paper, whenever we apply Lemma 2.6 to graphs or subtrees, it implicitly means that we apply the lemma to some equivalent trees obtained by Habiro's move 2 . 
The next result follows from Lemma 2.6 and [12, Proposition 2.7]. See also [4; 20].

Lemma 2.8 Let $G$ be a $Y_{k}$-tree in a 3-manifold $M$, and let $G_{+}$be a $Y_{k}$-tree obtained from $G$ by inserting a positive half twist in an edge. Then

$$
M_{G \cup \tilde{G}_{+}} \sim_{Y_{k+1}} M,
$$

where $\widetilde{G}_{+}$is obtained from $G_{+}$by an isotopy so that it is disjoint from $G$.

\subsection{The IHX relation for $Y_{k}$-graphs}

We have the following version of the IHX relation for $Y_{k}$-graphs.

Lemma 2.9 Let $I, H$ and $X$ be three $Y_{k}$-graphs in a 3-manifold $M$, which are identical except in a 3-ball where they look as depicted in Figure 2.8. Then

$$
M_{I} \sim_{Y_{k+1}} M_{H \cup \tilde{X}},
$$

where $\tilde{X}$ is obtained from $X$ by an isotopy so that it is disjoint from $H$.
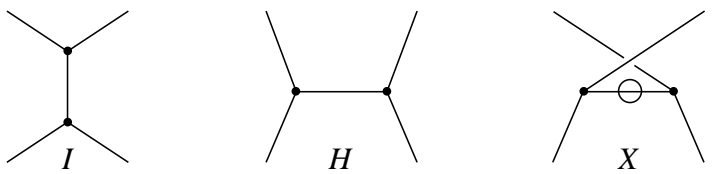

Figure 2.8: The three $Y_{k}$-graphs $I, H$ and $X$

Various similar statements appear in the literature. For example, an IHX relation is proved in [4] at the level of finite type invariants, in [3] for $C_{n}$-trees (see also [8]), and in [20, pages 397-398] for $Y_{n}$-graphs without leaves.

Proof For simplicity, we give the proof for the case of $Y_{2}$-trees. In the general case, the proof uses the same arguments as below, together with the zip construction ([12, Section 3], see also [3, Section 4.2]).

Consider the $Y_{2}$-tree $I$, and apply Lemma 2.4 at one of its nodes. Then, apply Habiro's move 11 so that we obtain the clasper $G_{1} \sim I$ depicted in Figure 2.9. By an isotopy and Habiro's move 7, $G_{1}$ is seen to be equivalent to the clasper $G_{2}$ of Figure 2.9. Consider the leaf of $G_{2}$ denoted by $f$ in the figure. By an application of Habiro's move 12 at $f$, followed by moves 7 and 11, we obtain the clasper $G_{3}$ of Figure 2.9. 


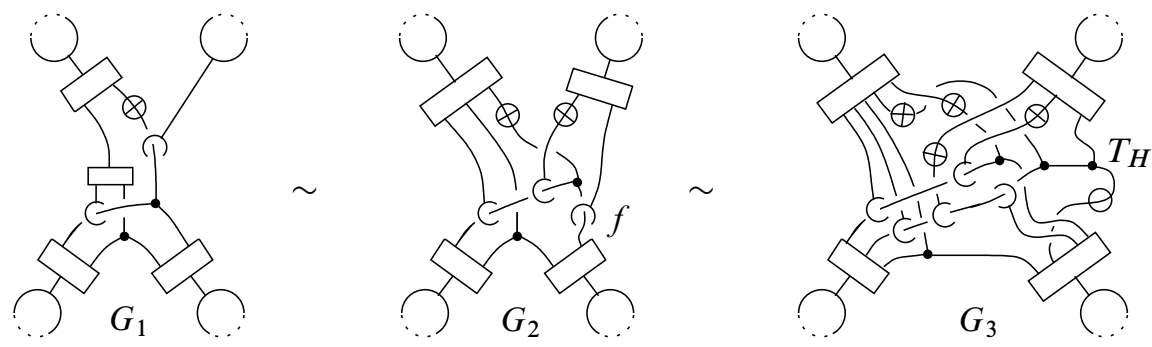

Figure 2.9

Observe that $G_{3}$ contains a $Y_{2}$-subtree $T_{H}$. By Habiro's move 6, Lemma 2.8 and Lemma 2.6 (1), we have

$$
M_{G_{3}} \sim_{Y_{3}} M_{H \cup G_{4}},
$$

where $G_{4}$ is the clasper depicted in Figure 2.10. Now, consider the leaf $f^{\prime}$ of $G_{4}$
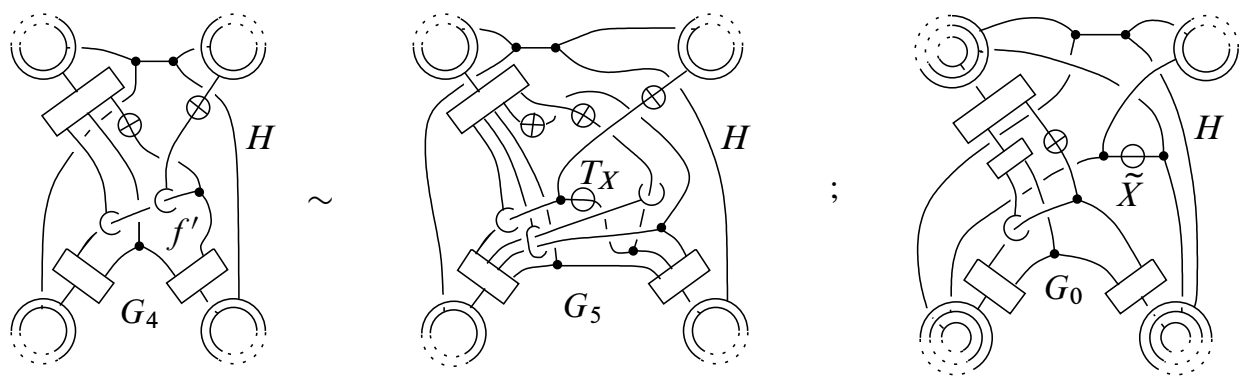

Figure 2.10

(see the figure). Apply Habiro's move 12 at $f^{\prime}$ and moves 7 and 11, just as we did previously for the clasper $G_{2}$. The resulting clasper $G_{5} \sim G_{4}$ contains a $Y_{2}$-subtree $T_{X}$ (see Figure 2.10). As above, we obtain by Lemmas 2.8 and 2.6 (1):

$$
M_{H \cup G_{5}} \sim_{Y_{3}} M_{H \cup \tilde{X} \cup G_{0}},
$$

where $G_{0}$ is represented in the right-hand side of Figure 2.10. By Habiro's moves 11 and 4, we obtain that $M_{H \cup \tilde{X} \cup G_{0}} \cong M_{H \cup \tilde{X}}$.

One can check the following slightly stronger version of Lemma 2.9 when $I, H$ and $X$ are three $Y_{k}$-trees (Note that Habiro's move 2 always allows us to have this condition satisfied).

Algebraic 8 Geometric Topology, Volume 6 (2006) 
Lemma 2.10 Let $I, H, X$ and $\tilde{X}$ be four $Y_{k}$-trees in a 3-manifold $M$ as in Lemma 2.9. Then

$$
M_{I} \sim_{Y_{k+2}} M_{H \cup \tilde{X} \cup F},
$$

where $F$ is a union of disjoint $Y_{k+1}$-trees. Each $Y_{k+1}$-tree $T$ in $F$ is obtained from either $H$ or $X$ by taking a parallel copy $f$ of one of its leaves, inserting a node $n$ in one of its edges, connecting $n$ and $f$ by an edge, and performing an isotopy so that $T$ is disjoint from $H, \tilde{X}$ and $F \backslash T$.

Consider for example the case of $Y_{2}$-trees, as in the proof of Lemma 2.9. We saw there that $M_{I} \cong M_{G_{3}} \sim_{Y_{3}} M_{H \cup G_{4}}$, where $G_{3}$ and $G_{4}$ are depicted in Figure 2.9 and 2.10. Observe that $H \cup G_{4}$ is obtained from $G_{3}$ by several Habiro's moves and three crossing changes between an edge of the $Y_{2}$-subtree $T_{H}$ and some leaf of $G_{3}$. So by (2) of Lemma 2.6 (and Habiro's move 5) one can check that

$$
M_{G_{3}} \sim Y_{4} M_{H \cup G_{4} \cup F^{\prime}}
$$

where $F^{\prime}$ consists of three $Y_{3}$-trees obtained as described in the statement of the Lemma. For similar reasons, (2) of Lemma 2.6 implies that the clasper $G_{5} \sim G_{4}$ depicted in Figure 2.10 satisfies $M_{H \cup G_{5}} \sim_{Y_{4}} M_{H \cup \tilde{X} \cup G_{0} \cup F^{\prime \prime}}$, where $F^{\prime \prime}$ is a union of $Y_{3}$-trees of the desired form. This implies Lemma 2.10 for $k=2$.

\section{Surgery along $Y_{n}$-trees with special leaves}

In this section, we study 3-manifolds obtained by surgery along $Y_{n}$-trees containing a particular type of leaves.

\section{1 m-special leaves}

Suppose we are given a clasper $G$ in a 3-manifold $M$.

Definition 3.1 Let $m \in \mathbf{Z}$. An $m$-special leaf with respect to $G$ is a leaf $f$ of $G$ which is an unknot bounding a disk $D$ in $M$ with respect to which it is $m$-framed, ${ }^{3}$ such that the interior of $D$ is disjoint from $G \backslash f$. D is called the bounding disk of $f$. Two bounding disks are required to be disjoint. A regular neighborhood of the union of $G$ and the bounding disks is called an s-regular neighborhood of $G$.

\footnotetext{
${ }^{3}$ Here, as in Definition 2.2, we regard a leaf as a knot with a framing.
} 
In particular, a 0 -special leaf with respect to $G$ is called a trivial leaf. If a $Y_{k}$-graph $G$ in $M$ contains a 0 -special leaf $f$ with respect to $G$, then $M_{G}$ is diffeomorphic to $M[12 ; 4]$.

In the rest of the paper, a special leaf is an $m$-special leaf for some unspecified integer $m .^{4}$ The mention 'with respect to' will be omitted when $G$ is clear from the context.

\subsection{Statement of the result}

Let $G$ be a $Y_{n}$-tree in a 3-manifold $M, n \geq 2$. It is well-known that, if $G$ contains a $(-1)$-special leaf, then

$$
M_{G} \sim_{Y_{n+1}} M .
$$

See [20, Lemma E.21] for a proof for $M=S^{3}$, which can be generalized to our context. See also [4, Lemma 4.9].

We obtain the following generalization.

Theorem 3.2 Let $G$ be a $Y_{n}$-tree in a 3-manifold $M$, with $n \geq 2$. Let $l$ denote the number of special leaves with respect to $G$. Then

(1) If $l<n$, then $M_{G} \sim_{Y_{n+l}} M$.

(2) If $l=n$, then $M_{G} \sim_{Y_{2 n-1}} M$.

(3) If $l>n$, then $M_{G} \sim_{Y_{2 n}} M$.

The proof is given in Section 3.6. In the next three subsections, we prove Theorem 3.2 in several important cases and provide a lemma which is used in Section 3.6.

\subsection{The case of a tree with one special leaf}

In this subsection, we prove Theorem 3.2 for $l=1$.

Lemma 3.3 Let $G$ be a $Y_{n}$-tree in a 3-manifold $M$, with $n \geq 2$. Suppose that $G$ contains an $m$-special leaf ; $m \in \mathbf{Z}$. Then $M_{G} \sim_{Y_{n+1}} M$.

Proof We first prove the lemma for all $m<0$, by induction. As recalled in Section 3.2, we already have the result for $m=-1$. Now consider a $Y_{n}$-tree $G$ in $M$ with an $m$-special leaf $f, m<0$. Denote by $G^{\prime}$ the clasper obtained by replacing $f$ by the union of a box $b$ and two edges $e_{1}$ and $e_{2}$ connecting $b$ respectively to a $(-1)-$ special

${ }^{4}$ Note that in some literature [4] the terminology 'special leaf' is used to denote a $(-1)$-special leaf. 
leaf $f_{1}$ and a $(m+1)$-special leaf $f_{2}$ (both leaves being special with respect to $G^{\prime}$ ). By Habiro's move 7, $G^{\prime} \sim G$. Denote by $G_{i}$ the $Y_{n}$-tree in $M$ obtained from $G$ by replacing $f$ by $f_{i}(i=1,2)$. By a zip construction, we have

$$
G^{\prime} \sim\left(G_{1} \cup P\right),
$$

where $P$ satisfies $P \sim G_{2}$. By (3-1) it follows that $M_{G} \sim_{Y_{n+1}} M_{G_{2}}$. The result then follows from the induction hypothesis.

Similarly, it would suffice to show the result for $m=1$ to obtain, by a similar induction, the result for all $m>0$. For this, consider the case $m=0$. In this case, $f$ is a trivial leaf and therefore $M_{G} \cong M$. The same construction as above, with a (-1)-special leaf $f_{1}$ and a 1 -special leaf $f_{2}$, shows that $M \sim_{Y_{n+1}} M_{G^{\prime}}$, where $G^{\prime}$ is a $Y_{n}$-tree in $M$ with a 1-special leaf. This concludes the proof.

\subsection{The case of a $Y_{2}$-tree}

In this section, we prove Theorem 3.2 for $n=2$. The proof mainly relies on the following lemma.

Lemma 3.4 Let $G$ be a $Y_{2}$-tree in a 3-manifold $M$ which contains two (-1)-special leaves which are connected to the same node. Then $M_{G} \sim_{Y_{4}} M$.

Proof Denote by $w$ the node of $G$ which is connected to the two special leaves. $w$ is connected by an edge to another node $v$. By applying Lemma 2.4 at $v, G$ is equivalent, in an $s$-regular neighborhood, to a clasper $G^{\prime}$ which is identical to $G$, except in a 3 -ball where it is as depicted in Figure 3.1 (a). There, the node $w^{\prime}$ corresponds to the node $w$ of $G$. By Lemma 2.3 and Habiro's move 6, we obtain the clasper depicted in Figure 3.1 (b), which is equivalent to the one depicted in Figure 3.1 (c) by three applications of Habiro's move 12, Lemma 2.5 and an isotopy. Denote by $G^{\prime \prime}$ this latter clasper. As the figure shows, $G^{\prime \prime}$ contains a $Y_{4}$-subtree $T$. Actually, $T$ is a ' $\operatorname{good}$ input subtree' of $G^{\prime \prime}$, in the sense of [12, Definition 3.13]. Denote by $\widetilde{G}^{\prime \prime}$ the clasper

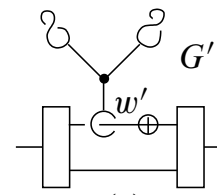

(a)

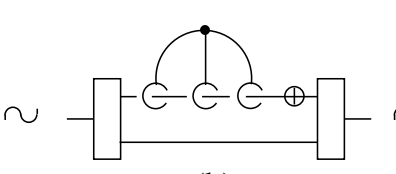

(b)

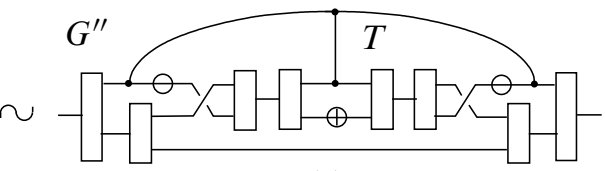

(c)

Figure 3.1 
obtained from $G^{\prime \prime}$ by inserting in each branch of $T$ a pair of small Hopf-linked leaves. By Habiro's move $2, \widetilde{G}^{\prime \prime} \sim G^{\prime}$. Denote by $\widetilde{T}$ the $Y_{4}$-tree of $\widetilde{G}^{\prime \prime}$ which corresponds to $T$. By an application of the zip construction, we obtain $M_{G^{\prime \prime}} \sim_{Y_{4}} M_{\widetilde{G^{\prime \prime}} \backslash \widetilde{T}}$. Further, it follows from Habiro's moves 3 and 4 that $\widetilde{G^{\prime \prime}} \backslash \tilde{T} \sim \varnothing$.

The following technical lemma will allow us to generalize Lemma 3.4 to arbitrary special leaves.

Lemma 3.5 Let $G$ be a $Y_{2}$-tree in a 3-manifold $M$ which contains two special leaves which are connected to the same node. Then

$$
M_{G} \sim Y_{4} M_{G_{1} \cup \widetilde{G}_{2}},
$$

where, for $i=1,2, G_{i}$ is obtained by replacing a $k$-special leaf of $G$ by a $k_{i}$-special leaf, such that $k_{1}+k_{2}=k$, and where $\widetilde{G}_{2}$ is obtained from $G_{2}$ by an isotopy so that it is disjoint from $G_{1}$.

Proof Denote respectively by $f$ and $f^{\prime}$ the $k$-special (resp. $k^{\prime}$-special) leaf of $G$, $k, k^{\prime} \in \mathbf{Z}$. Just as in the proof of Lemma 3.3, we can use Habiro's moves 7 and the zip construction to see that $G$ is equivalent, in an $s$-regular neighborhood, to the clasper $C_{1}$ of Figure 3.2, where $f_{1}$ is a $k_{1}$-special leaf and $f_{2}$ is a $k_{2}$-special leaf such that $k_{1}+k_{2}=k$. Consider the leaf of $C_{1}$ denoted by $F$ in the figure. By Habiro's move 12 at $F$, followed by two applications of Habiro's move 11 , we have $C_{1} \sim C_{2}$, where $C_{2}$ is represented in Figure 3.2.
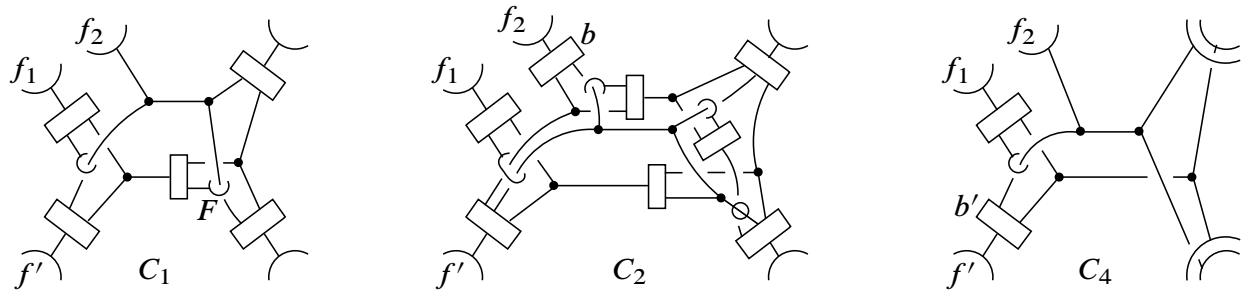

Figure 3.2

Consider the box $b$ of $C_{2}$ (see Figure 3.2). By applying Habiro's move 5 at $b, C_{2}$ is equivalent to a clasper containing a $Y_{3}$-subtree $T$ and a $Y_{1}$-subtree $T^{\prime}$ such that both $T$ and $T^{\prime}$ contain a copy of $f_{2}$. Denote by $C_{3}$ the clasper obtained by replacing these two (linked) copies of $f_{2}$ by two $k_{2}$-special leaves. By Lemma 2.6, we have $M_{C_{2}} \sim_{Y_{4}} M_{C_{3}}$. It follows from Lemma 3.3 and Habiro's move 5 that $M_{C_{3}} \sim_{Y_{4}} M_{C_{4}}$, 
where $C_{4}$ is as represented in Figure 3.2. By applying Habiro's move 5 at the box $b^{\prime}$, $C_{4}$ is equivalent to a clasper containing a $Y_{2}$-tree and a $Y_{2}$-subtree, each containing a copy of $f^{\prime}$. By Lemma 2.6, $M_{C_{4}} \sim_{Y_{4}} M_{C_{5}}$, where $C_{5}$ is obtained by replacing these two (linked) copies of $f^{\prime}$ in $C_{4}$ by two $k^{\prime}$-special leaves. The result then follows from an isotopy and Habiro's move 3.

We can now prove the case $n=2$ of Theorem 3.2.

Let $G$ be a $Y_{2}$-tree in a 3-manifold $M$ with $l$ special leaves. If $l=0$, then the result is obvious. If $l=1$, Lemma 3.3 implies that $M_{G} \sim_{Y_{3}} M$. If $l=2$, then $M_{G} \sim_{Y_{3}} M$ also follows from Lemma 3.3. It remains to prove the result when $l=3$ or 4 .

Let $k, k^{\prime} \in \mathbf{Z}$. Denote by $G_{k, k^{\prime}}$ a $Y_{2}$-tree in $M$ containing a $k$-special leaf $f$ and an $k^{\prime}$-special leaf $f^{\prime}$, both connected to the same node. Observe that it suffices to show that

$$
M_{G_{k, k^{\prime}}} \sim_{Y_{4}} M
$$

If $k=k^{\prime}=-1$, then (3-2) follows from Lemma 3.4. Now, let us fix $k^{\prime}=-1$. Then we can show by induction that (3-2) holds for all $k<-1$. Indeed, consider some integer $m<-1$, and consider $G_{m,-1}$ in $M$. By Lemma 3.5, we have

$$
M_{G_{m,-1}} \sim_{Y_{4}} M_{C_{1} \cup C_{2}},
$$

where $C_{1}$ contains two $(-1)$-special leaves connected to the same node, and where $C_{2}$ contains a (-1)-special leaf and an $m+1$-special leaf, both connected to the same node. By Lemma 3.4 and the induction hypothesis, we thus obtain $M_{G_{m,-1}} \sim_{Y_{4}} M$.

So we can now set $k^{\prime}$ to be any negative integer, and prove (3-2) for all $k<-1$, by strictly the same induction.

Similarly, it would suffice to show the result for $G_{1,1}$ to be able to prove (3-2) for all $k, k^{\prime}>0$. Consider $G_{0,-1}$ in $M$. In this case, $f$ is a trivial leaf and $M_{G_{0,1}} \cong M$. By applying Lemma 3.5 at $f$,

$$
M_{G_{0,1}} \cong M \sim_{Y_{4}} M_{G_{1} \cup G_{2}}
$$

where $G_{1}$ (resp. $G_{2}$ ) contains a $(-1)$-special leaf and a 1 -special (resp. $(-1)$-special) leaf, both connected to the same node. It follows from Lemma 3.3 that $M \sim_{Y_{4}} M_{G_{1}}$. This proves (3-2) for $k=1$ and $k=-1$. We obtain (3-2) for $k=k=1$ similarly, by applying Lemma 3.5 to $G_{0,1}$ in $M$.

Algebraic $8 \mathcal{G}$ Geometric Topology, Volume 6 (2006) 


\subsection{The cutting lemma.}

Let $G$ be a $Y_{n}$-tree in $M$, with $n \geq 3$. By inserting a pair of small Hopf-linked leaves in an edge of $G$, we obtain a $Y_{n_{1}}$-tree $G_{1}$ and a $Y_{n_{2}}$-tree $G_{2}$ such that $n_{1}+n_{2}=n$ and $G_{1} \cup G_{2} \sim G$ (by Habiro's move 2). See Figure 3.3.

Lemma 3.6 Let $i=1,2$. Suppose that, in a regular neighborhood $N_{i}$ of $G_{i}$, we have $\left(N_{i}\right)_{G_{i}} \sim_{Y_{k_{i}}} N_{i}$, with $k_{1} \geq 2$ and $k_{2} \geq 1$. Then

(1) $M_{G} \sim_{Y_{k_{1}+2}} M$, if $G_{2}$ is a $Y_{1}$-tree containing at least one special leaf with respect to $G_{1} \cup G_{2}$,

(2) $M_{G} \sim_{Y_{k_{1}+k_{2}}} M$, otherwise.

Proof Denote by $N$ an $s$-regular neighborhood of $G \sim G_{1} \cup G_{2}$. Consider a 3-ball $B$ in $M$ which intersects $N$ and $G_{1} \cup G_{2}$ as depicted in Figure 3.3 (a). Denote by

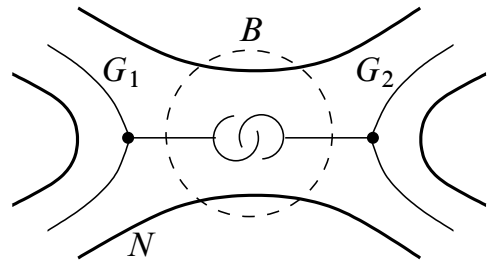

(a)

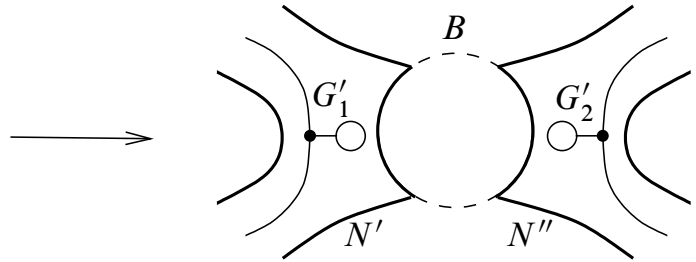

(b)

Figure 3.3

$N^{\prime}$ and $N^{\prime \prime}$ the two connected components of the closure of $N \backslash(B \cap N)$. By one crossing change and isotopy, we can homotop the two Hopf-linked leaves of $G_{1} \cup G_{2}$ into $N \backslash(B \cap N)$ so that, if $G_{1}^{\prime} \cup G_{2}^{\prime}$ denotes the resulting clasper, we have $G_{1}^{\prime} \subset N^{\prime}$ and $G_{2}^{\prime} \subset N^{\prime \prime}$. See Figure 3.3 (b). Each of $G_{1}^{\prime}$ and $G_{2}^{\prime}$ contains a trivial leaf with respect to $G_{1}^{\prime} \cup G_{2}^{\prime}$, so we have $G_{1}^{\prime} \cup G_{2}^{\prime} \sim \varnothing$ in $N$.

We now prove (1): suppose that $G_{2}$ contains one node and at least one special leaf with respect to $G_{1} \cup G_{2}$. Denote by $f$ the leaf of $G_{2}$ which forms a Hopf link with a leaf of $G_{1}$. By assumption, $G_{1}$ can be replaced by a $Y_{k_{1}}$-forest $F_{1}$ in an $s$-regular neighborhood $N_{1}$ so that $F_{1} \cup G_{2} \sim G$ in $N$. Consider a disk $d$ bounded by $f$ such that $d$ intersects transversally edges and leaves of components of $F_{1}$. By a sequence of crossing changes, we can homotop these edges and leaves into $N^{\prime} \subset N$ : the clasper $G^{\prime}$ obtained from $F_{1} \cup G_{2}$ by this homotopy satisfies $G^{\prime} \sim G_{1}^{\prime} \cup G_{2}^{\prime} \sim \varnothing$ in $N$. So it would suffice to show that $M_{F_{1} \cup G_{2}} \sim Y_{k_{1}+2} M_{G^{\prime}}$. 
By Lemma 2.6, we have $M_{F_{1} \cup G_{2}} \sim_{Y_{k_{1}+2}} M_{\tilde{F}_{1} \cup \tilde{G}_{2}}$, where $\tilde{F}_{1} \cup \widetilde{G}_{2}$ is obtained by 'homotoping' into $N^{\prime}$ all edges of $F_{1}$ and all $Y_{k}$-trees of $F_{1}$ with $k>k_{1}$. Denote by $\tilde{f}$ the leaf of $\widetilde{G}_{2}$ corresponding to $f$. There is a sequence of crossing changes

$$
\widetilde{F}_{1} \cup \widetilde{G}_{2}=C_{0} \mapsto C_{1} \mapsto C_{2} \mapsto \ldots \mapsto C_{p-1} \mapsto C_{p}=G^{\prime},
$$

where, for each $1 \leq k \leq p, C_{k}$ is obtained from $C_{k-1}$ by one crossing change between $\tilde{f}$ and a leaf $l$ of a $Y_{k_{1}}$-tree $T_{k}$ of $\tilde{F}_{1} .{ }^{5}$ By Lemma 2.6, we have $M_{C_{k}} \sim_{Y_{k_{1}+2}}$ $M_{C_{k-1} \cup H_{k}}$, where $H_{k}$ is a $Y_{k_{1}+1}$-tree obtained by connecting the edges of $\widetilde{G}_{2}$ and $T_{k}$ attached to $\tilde{f}$ and $l$ respectively. In particular, $H_{k}$ contains a special leaf with respect to $C_{k-1} \cup H_{k}$. So by Lemma 3.3, we have $M_{C_{k}} \sim_{Y_{k_{1}+2}} M_{C_{k-1}}$. It follows that $M_{\widetilde{F}_{1} \cup \widetilde{G}_{2}} \sim Y_{k_{1}+2} M_{G^{\prime}}$, which concludes the proof of (1).

The proof of (2) is simpler, and left to the reader. It uses exactly the same arguments as above, by considering the $Y_{k_{i}}$-forest $F_{i}(i=1,2)$ in an $s$-regular neighborhood $N_{i}$ of $G_{i}$ such that $F_{1} \cup F_{2} \sim G$ in $N$.

\subsection{Proof of Theorem 3.2}

Suppose that $G$ is a $Y_{n}$-tree in $M$ with $l$ special leaves ; $n \geq 2, l \geq 0$.

3.6.1 The case $l<n$ In this case, it is necessary to reduce the problem to linear trees. We have the following.

Claim 3.7 Let $1 \leq p \leq l$ be an integer. Pick two non-special leaves $f_{1}$ and $f_{2}$ of $G$. Then we have, by successive applications of the IHX relation,

$$
M_{G} \sim_{Y_{n+p}} M_{L_{p}}
$$

where $L_{p}$ is a union of disjoint linear $Y_{k}$-trees with $n \leq k \leq n+p-1$ such that

- the ends of each linear tree are parallel copies of $f_{1}$ and $f_{2}$,

- each $Y_{k}$-tree contains $(n+l-k)$ special leaves with respect to $L_{p}$.

Proof of the claim The claim is proved by induction on $p$. Observe that we can use the IHX relation to replace $T$ by a union $L_{1}$ of linear $Y_{k}$-trees whose ends are parallel copies of $f_{1}$ and $f_{2}$. Lemma 2.6 (1) ensures that each tree has $l$ special leaves with respect to $L_{1}$. This proves the case $p=1$. Now assume the claim for some $p \geq 1$ : $M_{T} \sim_{Y_{n+p}} M_{L_{p}}$, where $L_{p}$ is as described above. By assumption, this equivalence comes from Lemma 2.9, so we can apply Lemma 2.10. There exists a union $F$ of

\footnotetext{
${ }^{5}$ Here, abusing notations, we still denote by $\tilde{f}, \widetilde{G}_{2}$ and $\tilde{F}_{1}$ the corresponding elements in $C_{k}$, for all $k \geq 1$.
} 
disjoint (possibly non linear) $Y_{n+p}$-trees such that $M_{T} \sim_{Y_{n+p+1}} M_{L_{p} \cup F}$. For each tree $T$ in $F$, its $(n+p+2)$ leaves are obtained by taking the leaves of a $Y_{n+p-1}$-tree in $L_{p}$ and adding a parallel copy of one of them. If this additional leaf is a copy of a special leaf $f$ (with respect to $L_{p}$ ), the two (linked) copies of $f$ in $T$ are not special leaves with respect to $L_{p} \cup F$. This shows that each tree in $F$ contains at least $(l-p)$ special leaves with respect to $L_{p} \cup F$. Note that each such tree also contains (at least) a copy of $f_{1}$ and $f_{2}$. So by Lemma 2.9 we have $M_{L_{p} \cup F} \sim_{Y_{n+p+1}} M_{L_{p+1}}$, where $L_{p+1}$ is of the desired form.

It follows from Claim 3.7 that

$$
M_{T} \sim_{Y_{n+l}} M_{L}
$$

where $L$ is a union of linear $Y_{k}$-trees with $n \leq k \leq n+l-1$, each such linear $Y_{k}$-tree containing (at least) $(n+l-k)$ special leaves with respect to $L$, and whose ends are non-special leaves.

So it suffices to prove the case $l<n$ of Theorem 3.2 for linear $Y_{n}$-trees whose ends are non-special leaves. We proceed by induction on $n$.

For $n=2$, the statement follows from Section 3.4.

Now, assume that the statement holds true for all $k<n$, and consider a linear $Y_{n}$-tree $G$ whose ends are two non-special leaves. Insert a pair of small Hopf-linked leaves in an edge of $G$ such that it produces a union of two linear trees $G_{1} \cup G_{2} \sim G$ with $\operatorname{deg} G_{1}=n_{1}$ and $\operatorname{deg} G_{2}=n_{2}$. Denote respectively by $l_{1}$ and $l_{2}$ the number of special leaves with respect to $G_{1} \cup G_{2}$ in $G_{1}$ and $G_{2}$. We have $n_{1}+n_{2}=n$ and $l_{1}+l_{2}=l$. Denote also by $N_{1}$ an $s$-regular neighborhood of $G_{1}$.

- If we can choose $n_{2}=1$ and $l_{2}=1$, then $n_{1}=n-1$ and $l_{1}=l-1$. So $l_{1}<n_{1}$ and by the induction hypothesis we have $\left(N_{1}\right)_{G_{1}} \sim Y_{n+l-2} N_{1}\left(G_{1}\right.$ is indeed linear). As $G_{2}$ contains one special leaf with respect to $G_{1} \cup G_{2}$, we obtain the result by Lemma $3.6(1)$.

- Otherwise, then $l<n-1$, and we can choose $G_{2}$ such that $n_{2}=1$ and $l_{2}=0$ (that is, $G_{2}$ contains one node connected to 2 non-special leaves). As $l_{1}=l<n_{1}=n-1$, we have $\left(N_{1}\right)_{G_{1}} \sim_{Y_{n+l-1}} N_{1}$ (by the induction hypothesis), and the result follows from Lemma 3.6 (2).

This completes the proof of the case $l<n$.

Algebraic ${ }^{6} \mathcal{G}$ Geometric Topology, Volume 6 (2006) 
3.6.2 The case $l \geq n \quad$ The case $l=n$ follows immediately from the case $l=n-1$, by regarding one of the special leaves as a leaf.

We prove the case $l=n+1$ by induction on the degree $n$. The case $n=2$ was proved in Section 3.4. Consider a $Y_{n}$-tree $G$ with $l \geq n$ special leaves. As in Section 3.6.1, insert a pair of Hopf-linked leaves in an edge of $G$ so that we obtain a union of two trees $G_{1} \cup G_{2} \sim G$ with $\operatorname{deg} G_{1}=n-1$ and $\operatorname{deg} G_{2}=1$. Denote respectively by $l_{1}$ and $l_{2}$ the number of special leaves with respect to $G_{1} \cup G_{2}$ in $G_{1}$ and $G_{2}$. There are two cases, depending on whether $l_{2}=1$ or 2 .

- If $l_{2}=1$, then $l_{1}=n=n_{1}+1$, and thus, by the induction hypothesis we have $\left(N_{1}\right)_{G_{1}} \sim_{Y_{2 n-3}} N_{1}$ in an $s$-regular neighborhood $N_{1}$ of $G_{1}$. The result follows from Lemma $3.6(1)$.

- If $l_{2}=2$, then $l_{1}=n-1=n_{1}$. It thus follows from the case $l=n$ of Theorem 3.2 that $\left(N_{1}\right)_{G_{1}} \sim Y_{2 n-3} N_{1}$ in an $s$-regular neighborhood $N_{1}$ of $G_{1}$. The result then follows as above from Lemma 3.6 (1).

The case $l=n+2$ follows from the case $l=n+1$ by regarding one of the special leaves as a leaf.

\subsection{Some special cases for Theorem 3.2}

We have the following improvement of Theorem 3.2 for linear trees having only $(-1)$-special leaves.

Proposition 3.8 Let $G$ be a linear $Y_{n}$-tree in a 3-manifold $M, n \geq 2$, such that all its leaves are (-1)-special leaves. Then in an $s$-regular neighborhood $N$ of $G$ (which is a 3-ball in $M$ ) we have

$$
N_{G} \sim_{Y_{2 n+1}} N_{\Theta_{n}},
$$

where $\Theta_{n}$ is the connected $Y_{2 n}$-graph without leaves depicted in Figure 3.4

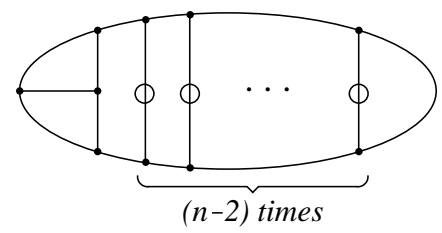

Figure 3.4: The $Y_{2 n}-$ graph $\Theta_{n}$ 
Remark 3.9 Note that " $\sim_{Y_{2 n+1}}$ " in Proposition 3.8 can be replaced by " $\sim_{Y_{2 n+2}}$ ". This follows from the fact that if two integral homology balls are $Y_{2 n+1}$-equivalent then they are $Y_{2 n+2}$-equivalent $(n>1)$.

The proof of Proposition 3.8 uses rather involved calculus of claspers, and is therefore postponed to Section 7. Note that this result is not needed for the rest the paper. A reader who is not too comfortable with claspers (but who nevertheless reached this point) may thus safely skip this proof.

Also, one can check that if $G$ is a $Y_{n}$-tree in a 3 -manifold $M$ with $n$ special leaves, we have

$$
M_{G} \sim_{Y_{2 n}} M
$$

in the two following situations:

- $G$ contains a $2 k$-special leaf, for some integer $k$.

- The homology class in $H_{1}(M ; \mathbf{Z} / 2 \mathbf{Z})$ of a non-special leaf of $G$ is zero. ${ }^{6}$ In particular, (3-3) always holds if $M=S^{3}$.

\section{$4 Y_{k}$-equivalence for 3-manifolds obtained by surgery along Brunnian links}

In this section, we prove Theorems 1.1 and 1.2. The proofs use a characterization of Brunnian links in terms of claspers due to Habiro, and independently to Miyazawa and Yasuhara, which involves the notion of $C_{k}^{a}$-equivalence. Let us first recall from [11] the definition and some properties of this equivalence relation.

\section{1 $C_{k}^{a}$-equivalence}

Definition 4.1 Let $L$ be an $m$-component link in a 3-manifold $M$. For $k \geq m-1$, a $C_{k}^{a}$-tree for $L$ in $M$ is a $C_{k}$-tree $T$ for $L$ in $M$, such that

(1) all the strands intersecting a given disk-leaf of $T$ are from the same component of $L$,

(2) $T$ intersects all the components of $L$.

\footnotetext{
${ }^{6}$ This fact was pointed out to the author by Kazuo Habiro.
} 
A (simple) $C_{k}^{a}$-forest $L$ is a clasper consisting only of (simple) $C_{k}^{a}$-tree for $L$.

A $C_{k}^{a}$-move on a link is surgery along a $C_{k}^{a}$-tree. The $C_{k}^{a}$-equivalence is the equivalence relation on links generated by $C_{k}^{a}$-moves.

The main tool in the proofs of Theorems 1.1 and 1.2 is the following.

Theorem 4.2 $[11 ; 18]$ Let $L$ be an $(n+1)$-component link in $S^{3} . L$ is Brunnian if and only if it is $C_{n}^{a}$-equivalent to the $(n+1)$-component unlink $U$.

In the proof of Theorem 1.2, we will also need the next result.

Theorem 4.3 ([18], see also [13]) Two $(n+1)$-component Brunnian links in $S^{3}$ are link-homotopic if and only if they are $C_{n+1}^{a}$-equivalent.

Note that this statement does not appear explicitly in [18]. However, it is implied by the proof of [18, Theorem 3]. An alternative proof was given subsequently by Habiro and the author [13].

\subsection{Proof of Theorem 1.1}

Let $m=\left(m_{1}, \ldots, m_{n+1}\right) \in \mathbf{Z}^{n+1}, n \geq 2$ and let $L$ be an $(n+1)$-component Brunnian link in a 3 -manifold $M$. By Theorem 4.2, $L$ is $C_{n}^{a}$-equivalent to an $(n+1)-$ component unlink $U$ in $M$. So by [11, Lemma 7] there exists a simple $C_{n}^{a}$-forest $F=T_{1} \cup \ldots \cup T_{p}$ for $U$ such that $L \cong U_{F}$. We thus have

$$
M_{(L, m)} \cong M_{G_{m}(F)},
$$

where $G_{m}(F)$ is the clasper obtained from $F$ by performing $\frac{1}{m_{i}}$-framed surgery along the $i^{\text {th }}$ component $U_{i}$ of $U$ for all $1 \leq i \leq n+1$. Indeed, $\frac{1}{m_{i}}$-surgery along an unknot does not change the diffeomorphism type of $M$, and can be regarded as a move on claspers in $M$. Observe that $\frac{1}{m_{i}}$-surgery along $U_{i}$ turns each disk-leaf of $F$ intersecting $U_{i}$ into a $\left(-m_{i}\right)$-framed unknot (here, we forget the bounding disk). Thus $\frac{1}{m}$-surgery along $U$ turns each $C_{n}^{a}$-tree $T_{j}$ of $F$ into a $Y_{n-1}$-tree $G_{j}$ in $M$. However, the $(n+1)$ corresponding leaves of $G_{j}$ might not be special leaves with respect to $G_{m}(F)$, as they can be linked with the leaves of other components of $G_{m}(F)$. Lemma 2.6 (1) can be used to unlink these leaves 'up to $Y_{2 n-2}$-equivalence'. Namely, Lemma 2.6 implies that $M_{G_{m}(F)} \sim_{Y_{2 n-2}} M_{\widetilde{G}_{m}(F)}$, where $\widetilde{G}_{m}(F)$ is a union of $Y_{n-1}$-trees, each containing $(n+1)$ special leaves with respect to $\widetilde{G}_{m}(F)$. The result then follows from Theorem 3.2. 


\subsection{Proof of Theorem 1.2}

Let $L$ and $L^{\prime}$ be two link-homotopic $(n+1)$-component Brunnian links in $M$, and let $U$ denote an $(n+1)$-component unlink $U$ in $M$. By Theorems 4.2 and 4.3 , and [11, Lemma 7], there exists a simple $C_{n+1}^{a}$-forest $F=T_{1} \cup \ldots \cup T_{p}$ and a simple $C_{n}^{a}$-forest $F^{\prime}=T_{1}^{\prime} \cup \ldots \cup T_{q}^{\prime}$ for $U$ such that $L^{\prime} \cong U_{F^{\prime}}$ and $L \cong U_{F \cup F^{\prime}}$.

For all $j$, denote by $G_{j}^{\prime}$ (resp. $G_{j}$ ) the $Y_{n-1}$-tree (resp. $Y_{n}$-tree) obtained from $T_{j}^{\prime}$ (resp. $T_{j}$ ) by $\frac{1}{m}$-surgery along $U$. By Lemma 2.6,

$$
M_{(L,+1)} \sim_{Y_{2 n-1}} M_{G_{1}^{\prime} \cup \ldots \cup G_{q}^{\prime}} \sharp S_{G_{1}}^{3} \sharp \ldots \sharp S_{G_{p}}^{3} \cong M_{\left(L^{\prime},+1\right)} \sharp S_{G_{1}}^{3} \sharp \ldots \sharp S_{G_{p}}^{3} .
$$

So proving that $S_{G_{i}}^{3} \sim_{Y_{2 n-1}} S^{3}$ for all $1 \leq i \leq p$ would imply the theorem.

By strictly the same arguments as in Section 4.2, the $Y_{n}$-tree $G_{i}$ contains at least $n$ special leaves, for all $1 \leq i \leq p$. So Theorem 3.2 implies that $S_{G_{i}}^{3} \sim_{Y_{2 n-1}} S^{3}$.

\section{Trivalent diagrams and Goussarov-Vassiliev invariants for Brunnian links}

In this section, we recall some results proved by Habiro and the author in a previous paper [14]. These, together with the two theorems shown in Section 4, will allow us to prove Theorem 1.3 in the next section.

\subsection{Trivalent diagrams}

A trivalent diagram is a finite graph with trivalent vertices, each vertex being equipped with a cyclic order on the three incident edges. The degree of a trivalent diagram is half the number of vertices.

For $k \geq 0$, let $\mathcal{A}_{k}(\varnothing)$ denote the $\mathbf{Z}$-module generated by trivalent diagrams of degree $k$, subject to the $A S$ and IHX relations, see Figure 5.1.

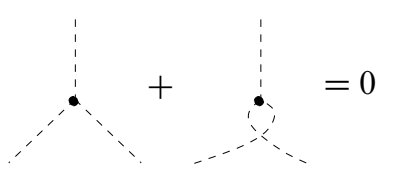

AS

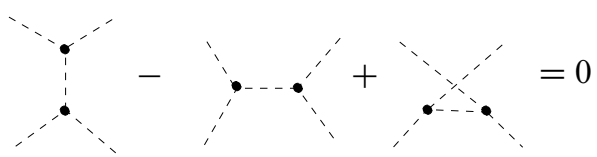

IHX

Figure 5.1: The AS and IHX relations

Denote by $\mathcal{A}_{k}^{c}(\varnothing)$ the $\mathbf{Z}$-submodule of $\mathcal{A}_{k}(\varnothing)$ generated by connected trivalent diagrams. 


\subsection{The Brunnian part of the Goussarov-Vassiliev filtration}

Denote by $\mathbf{Z} \mathcal{L}(n)$ the free $\mathbf{Z}$-module generated by the set of isotopy classes of $n-$ component links in $S^{3}$, and denote by $J_{k}(n)$ the $\mathbf{Z}$-submodule of $\mathbf{Z} \mathcal{L}(n)$ generated by elements of the form

$$
\left[L ; C_{1}, \ldots, C_{p}\right]:=\sum_{S \subseteq\left\{C_{1}, \ldots, C_{p}\right\}}(-1)^{|S|} L_{S},
$$

where $L$ is an $n$-component link in $S^{3}$, and where the $C_{i}(1 \leq i \leq p)$ are disjoint $C_{k_{i}}$-trees for $L$ such that $k_{1}+\ldots+k_{p}=k$. The sum runs over all the subsets $S$ of $\left\{C_{1}, \ldots, C_{p}\right\}$ and $|S|$ denotes the cardinality of $S$. The descending filtration

$$
\mathbf{Z} \mathcal{L}(n)=J_{0}(n) \supset J_{1}(n) \supset J_{2}(n) \supset \ldots
$$

coincides with the Goussarov-Vassiliev filtration [12].

Denote by $\bar{J}_{k}(n)$ the graded quotient $J_{k}(n) / J_{k+1}(n)$.

Definition 5.1 The Brunnian part $\operatorname{Br}\left(\bar{J}_{2 n}(n+1)\right)$ of the $2 n^{\text {th }}$ graded quotient $\bar{J}_{2 n}(n+1)$ is the $\mathbf{Z}$-submodule generated by elements $[L-U]_{J_{2 n+1}}$ where $L$ is an $(n+1)$-component Brunnian link.

As outlined in [13, Section 7], $\operatorname{Br}\left(\bar{J}_{2 n}(n+1)\right)$ is spanned over $\mathbf{Z}$ by elements

$$
\frac{1}{2}\left[U ; T_{\sigma} \cup \tilde{T}_{\sigma}\right] \text { and }\left[U ; T_{\sigma} \cup \widetilde{T}_{\sigma^{\prime}}\right], \quad \text { for } \sigma \neq \sigma^{\prime} \in S_{n-1},
$$

where, for all $\sigma, \sigma^{\prime}$ in the symmetric group $S_{n-1}, T_{\sigma}$ is the simple linear $C_{n}^{a}$-tree for the $(n+1)$-component unlink $U$ depicted in Figure 5.2, and $\widetilde{T}_{\sigma^{\prime}}$ is obtained from $T_{\sigma^{\prime}}$ by a small isotopy so that it is disjoint from $T_{\sigma}$. (Here $\frac{1}{2}\left[U ; T_{\sigma} \cup \widetilde{T}_{\sigma}\right]$ means an element $x \in \operatorname{Br}\left(\bar{J}_{2 n}(n+1)\right)$ such that $2 x=\left[U ; T_{\sigma} \cup \widetilde{T}_{\sigma}\right]$. Existence of such an element is shown in [13].)

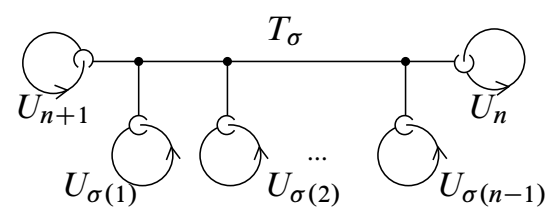

Figure 5.2: The simple linear $C_{n}^{a}$-tree $T_{\sigma}$ 


\subsection{The map $h_{n}: \mathcal{A}_{n-1}^{c}(\varnothing) \rightarrow \operatorname{Br}\left(\bar{J}_{2 n}(n+1)\right)$}

Connected trivalent diagrams allow us to describe the structure of $\operatorname{Br}\left(\bar{J}_{2 n}(n+1)\right)$. For $n \geq 2$, we have a map

$$
h_{n}: \mathcal{A}_{n-1}^{c}(\varnothing) \longrightarrow \bar{J}_{2 n}(n+1)
$$

defined as follows. Given a connected trivalent diagram $\Gamma \in \mathcal{A}_{n-1}^{c}(\varnothing)$, insert $n+1$ ordered copies of $S^{1}$ in the edges of $\Gamma$, in an arbitrary way. The result is a strict unitrivalent graphs $D_{\Gamma}$ of degree $2 n$ on the disjoint union of $(n+1)$ copies of $S^{1}$ (see [1]). Next, 'realize' this unitrivalent graph by a graph clasper. Namely, replace each univalent vertex (resp. trivalent vertex, edge) of $D_{\Gamma}$ with a disk-leaf (resp. node, edge), these various subsurfaces being connected as prescribed by the graph $D_{\Gamma}$. Denote by $C\left(D_{\Gamma}\right)$ the resulting graph clasper for the $(n+1)$-component unlink $U$. Then

$$
h_{n}(\Gamma):=\left[U-U_{C\left(D_{\Gamma}\right)}\right]_{J_{2 n+1}} \in \bar{J}_{2 n}(n+1) .
$$

For $n \geq 3$, the image of $h_{n}$ is the Brunnian part $\operatorname{Br}\left(\bar{J}_{2 n}(n+1)\right)$ of $\bar{J}_{2 n}(n+1)$, and

$$
h_{n} \otimes \mathbf{Q}: \mathcal{A}_{n-1}^{c}(\varnothing) \otimes \mathbf{Q} \longrightarrow \operatorname{Br}\left(\bar{J}_{2 n}(n+1)\right) \otimes \mathbf{Q}
$$

is an isomorphism.

\section{Finite type invariants of integral homology spheres}

\subsection{The Ohtsuki filtration for integral homology spheres}

Let $\mathcal{M}$ denote the free $\mathbf{Z}$-module generated by the set of orientation-preserving homeomorphism classes of integral homology spheres. The definition of the Ohtsuki filtration uses algebraically split, unit-framed links. For the purpose of the present paper, it is however more convenient to use a definition using claspers, due to Goussarov and Habiro [4; 7; 12]. For $k \geq 0$, let $\mathcal{M}_{k}$ denote the $\mathbf{Z}$-submodule of $\mathcal{M}$ generated by elements of the form

$$
\left[M ; G_{1}, \ldots, G_{p}\right]:=\sum_{S \subseteq\left\{G_{1}, \ldots, G_{p}\right\}}(-1)^{|S|} M_{S},
$$

where $M$ is an integral homology sphere, and where the $G_{i}(1 \leq i \leq p)$ are disjoint $Y_{k_{i}}$-graphs in $M$ such that $k_{1}+\ldots+k_{p}=k$. The sum runs over all the subsets $S$ of $\left\{G_{1}, \ldots, G_{p}\right\}$ and $|S|$ denotes the cardinality of $S$.

The descending filtration of $\mathbf{Z}$-submodules

$$
\mathcal{M}=\mathcal{M}_{0} \supset \mathcal{M}_{1} \supset \mathcal{M}_{2} \supset \ldots
$$

Algebraic $8 \mathcal{G}$ Geometric Topology, Volume 6 (2006) 
is equal to the Ohtsuki filtration after re-indexing and tensoring by $\mathbf{Z}[1 / 2][4 ; 7 ; 12]$. Another alternative definition was previously given by Garoufalidis and Levine using 'blinks' [5].

\subsection{The connected part of the Ohtsuki filtration}

Let $\overline{\mathcal{M}}_{2 k}$ denote the graded quotient $\mathcal{M}_{2 k} / \mathcal{M}_{2 k+1}$.

Definition 6.1 The connected part $\operatorname{Co}\left(\overline{\mathcal{M}}_{2 k}\right)$ of $\overline{\mathcal{M}}_{2 k}$ is the $\mathbf{Z}$-submodule of $\overline{\mathcal{M}}_{2 k}$ generated by elements $\left[S^{3} ; G\right]_{\mathcal{M}_{2 k+1}}$ where $G$ is a $Y_{2 k}$-graph (in particular, $G$ is connected).

For $k \geq 1$, there is a well-defined surgery map

$$
\varphi_{k}: \mathcal{A}_{k}(\varnothing) \longrightarrow \overline{\mathcal{M}}_{2 k},
$$

which maps each trivalent diagram $\Gamma=\Gamma_{1} \cup \ldots \cup \Gamma_{p}$ to $\left[S^{3} ; G_{\Gamma_{1}}, \ldots, G_{\Gamma_{p}}\right]$, where $G_{\Gamma_{i}}$ is a connected clasper obtained by 'realizing' the diagram $\Gamma_{i}$ in $S^{3}$ as depicted in Figure 6.1. The image $\varphi_{k}(\Gamma)$ of a degree $k$ trivalent diagram $\Gamma$ in $\overline{\mathcal{M}}_{2 k}$ by $\varphi_{k}$ does not depend on the embeddings $G_{\Gamma_{i}}$ in $S^{3}$ ([12], see also [20, page 320]). Note that $\varphi_{k}$ is a reconstruction, using claspers, of a map defined previously by Garoufalidis and Ohtsuki [6]. The homomorphism $\varphi_{k} \otimes \mathbf{Z}[1 / 2]$ is surjective, and it is an isomorphism

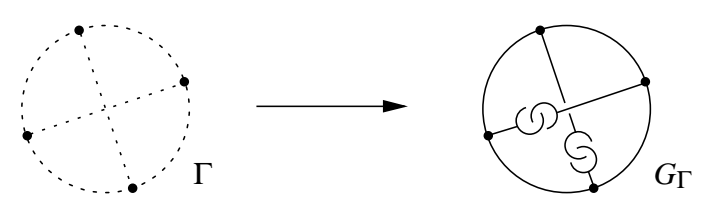

Figure 6.1: Realizing a trivalent diagram in $S^{3}$

when tensoring by $\mathbf{Q}$, with inverse given by the LMO invariant [15].

It can be easily checked using the arguments of [4] that $\varphi_{k}\left(\mathcal{A}_{k}^{c}(\varnothing)\right)=\operatorname{Co}\left(\overline{\mathcal{M}}_{2 k}\right)$. We thus have an isomorphism

$$
\varphi_{k} \otimes \mathbf{Q}: \mathcal{A}_{k}^{c}(\varnothing) \otimes \mathbf{Q} \stackrel{\simeq}{\longrightarrow} \operatorname{Co}\left(\overline{\mathcal{M}}_{2 k}\right) \otimes \mathbf{Q}
$$

induced by the surgery map $\varphi_{k}$.

Algebraic ${ }^{6} \mathcal{G}$ Geometric Topology, Volume 6 (2006) 


\subsection{The $\operatorname{map} \alpha_{k}: \operatorname{Co}\left(\overline{\mathcal{M}}_{2 k}\right) \longrightarrow \overline{\mathcal{S}}_{2 k}$}

Let $\mathcal{S}_{k}$ denote the set of integral homology spheres which are $Y_{k}$-equivalent to $S^{3}$, and denote by $\overline{\mathcal{S}}_{k}$ the quotient $\mathcal{S}_{k} / \sim_{Y_{k+1}}$. The connected sum induces an abelian group structure on $\overline{\mathcal{S}}_{k}$.

As recalled in the introduction, $\overline{\mathcal{S}}_{2 k+1}=0$ for all $k \geq 1 . \overline{\mathcal{S}}_{2 k}$ is generated by the elements $S_{G}^{3}$, where $G$ is a $Y_{2 k}$-graph in $S^{3}$ (for $k=0$, we have $\overline{\mathcal{S}}_{1}=\mathbf{Z} / 2 \mathbf{Z}$ ). There is a surjective homomorphism of abelian groups

$$
\phi_{k}: \mathcal{A}_{k}^{c}(\varnothing) \longrightarrow \overline{\mathcal{S}}_{2 k}
$$

defined by $\phi_{k}(\Gamma):=\left[S_{G_{\Gamma}}^{3}\right]_{Y_{2 k+1}}$, where $G_{\Gamma}$ is a topological realization of the diagram $\Gamma$ as in the definition of $\varphi_{k}$ (see Figure 6.1). It is well known that $\phi_{k}$ is well-defined (see the proof of [20, Theorem E.20]).

The map $\phi_{k}$ is an isomorphism over the rationals. This is shown by using the primitive part of the LMO invariant $z^{L M O}$ [20, pages 329-330].

Let

$$
\alpha_{k}: \operatorname{Co}\left(\overline{\mathcal{M}}_{2 k}\right) \longrightarrow \overline{\mathcal{S}}_{2 k}
$$

be the map defined by

$$
\alpha_{k}\left(\left[S^{3} ; G\right]_{\mathcal{M}_{2 k+1}}\right)=\left[S_{G}^{3}\right]_{Y_{2 k+1}} .
$$

The fact that $\alpha_{k}$ is well-defined follows from standard arguments of clasper theory, and is well known to experts.

The following is clear from the above definitions.

Lemma 6.2 The following diagram commutes for all $k \geq 1$ :

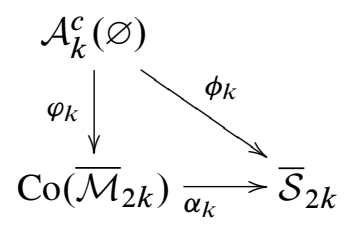

As a consequence, $\alpha_{k}$ is an isomorphism over the rationals.

Algebraic ${ }^{6} \mathcal{G}$ Geometric Topology, Volume 6 (2006) 


\subsection{The map $\lambda_{n}$}

For simplicity, we work over the rationals in the rest of this section.

Let $n \geq 2$. Denote by $B_{n+1}$ the set of isotopy classes of $(n+1)$-component Brunnian links in $S^{3}$. Define a linear map

$$
\tilde{\lambda}_{n}: \mathbf{Q} B_{n+1} \rightarrow \mathcal{M}
$$

by assigning each element $L \in B_{n+1}$ to $S_{(L, 1)}^{3}$. Note that $\tilde{\lambda}_{n}$ is well-defined, as $S_{(L,+1)}^{3}$ is an integral homology sphere for all $L \in B_{n+1}$.

Denote by $\mathbf{I}$ the submodule of $\mathbf{Q} B_{n+1}$ generated by element $\left(L-L^{\prime}\right)$ such that $\tilde{\lambda}_{n}\left(L-L^{\prime}\right)$ is in $\mathcal{M}_{2 n-1}$. The following follows immediately from [11] and Theorem 1.2 .

Lemma 6.3 Let $L$ and $L^{\prime}$ be two link-homotopic (or $C_{n+1}^{a}$-equivalent) $(n+1)-$ component Brunnian links. Then $L-L^{\prime} \in \mathbf{I}$.

Note that two link-homotopic $(n+1)$-component Brunnian links satisfy $L-L^{\prime} \in$ $J_{2 n+1}(n+1)$ [13, Proposition 7.1]. We generalize Lemma 6.3 as follows.

Proposition 6.4 Let $L, L^{\prime}$ be two $(n+1)$-component Brunnian links in $S^{3}$ such that $L-L^{\prime} \in J_{2 n+1}(n+1)$. Then $L-L^{\prime} \in \mathbf{I}$.

Proof Let $B$ be an $(n+1)$-component Brunnian link in $S^{3}$. By [13, Section 5], we have $B \sim C_{n+1}^{a} U_{F}$, where $F=T_{1} \cup \ldots \cup T_{m}$ is a simple $C_{n}^{a}$-forest $F$ for $U$ in $S^{3}$ such that, for all $1 \leq i \leq p$, we have $T_{i}=T_{\sigma_{i}}$ for some $\sigma_{i} \in S_{n-1}$ (see Figure 5.2 for the definition of $T_{\sigma_{i}}$ ). By Lemma 6.3 we thus have

$$
B \equiv U_{F} \bmod \mathbf{I} .
$$

Observe that we have the equality

$$
U_{F}=\sum_{F^{\prime} \subseteq F}(-1)^{\left|F^{\prime}\right|}\left[U ; F^{\prime}\right]
$$

For all $F^{\prime} \subseteq F$, denote by $G\left(F^{\prime}\right)$ the clasper obtained in $S^{3}$ by performing $(+1)-$ framed surgery along $U$. As in Section 4.2, we have $\tilde{\lambda}_{n}\left(U_{F}\right)=S_{\left(U_{F},+1\right)}^{3} \cong S_{G(F)}^{3}$. As each $C_{n}^{a}$-tree in $F^{\prime}$ is turned into a $Y_{n-1}$-tree of $S^{3}$ by this operation, we have $\tilde{\lambda}_{n}\left(\left[U ; F^{\prime}\right]\right)=\left[S^{3} ; G\left(F^{\prime}\right)\right] \in \mathcal{M}_{(n-1) .\left|F^{\prime}\right|}$. In particular, $\tilde{\lambda}_{n}\left(\left[U ; F^{\prime}\right]\right) \in \mathcal{M}_{2 n-2}$ for all $F^{\prime}$ with $\left|F^{\prime}\right| \geq 3$. It follows that

$$
B \equiv \sum_{F^{\prime} \subseteq F /\left|F^{\prime}\right| \leq 2}(-1)^{\left|F^{\prime}\right|}\left[U ; F^{\prime}\right] \bmod \mathbf{I} .
$$

Algebraic 83 Geometric Topology, Volume 6 (2006) 
By strictly the same arguments as in the proof of [13, Theorem 7.4], one can check that, for every $\sigma \in S_{n-1},\left[U ; T_{\sigma}\right] \equiv \frac{1}{2}\left[U ; T_{\sigma}, \widetilde{T}_{\sigma}\right] \bmod \mathbf{I}$. It follows that

$$
B \equiv U+\frac{1}{2} \sum_{1 \leq i \leq m}\left[U ; T_{\sigma_{i}}, \widetilde{T}_{\sigma_{i}}\right]+\sum_{1 \leq i \neq j \leq m}\left[U ; T_{\sigma_{i}}, \widetilde{T}_{\sigma_{j}}\right] \bmod \mathbf{I}
$$

It follows that $L-L^{\prime}$ is equal, modulo $\mathbf{I}$, to a linear combination of the form $\left(\alpha_{\sigma, \sigma^{\prime}} \in \mathbf{Q}\right)$

$$
\sum_{\sigma, \sigma^{\prime} \in S_{n-1}} \alpha_{\sigma, \sigma^{\prime}}\left[U ; T_{\sigma}, \widetilde{T}_{\sigma^{\prime}}\right]
$$

By assumption, $L-L^{\prime} \in J_{2 n+1}(n+1)$. So (6-1) vanishes in $\operatorname{Br}\left(\bar{J}_{2 n}(n+1)\right)$, and is thus mapped by $h_{n}^{-1}$ onto a linear combination of connected trivalent diagrams which vanishes in $\mathcal{A}_{n-1}^{c}(\varnothing)$. (6-1) is thus a linear combination of terms of the following two types.

(1) (AS) $\left[U ; T_{1}, T_{2}\right]+\left[U ; T_{1}^{\prime}, T_{2}^{\prime}\right]$, where $T_{1} \cup T_{2}$ and $T_{1}^{\prime} \cup T_{2}^{\prime}$ differ by the cyclic order of the three edges attached to a node.

(2) (IHX) $\left[U ; T_{1}, T_{2}\right]+\left[U ; T_{1}^{\prime}, T_{2}^{\prime}\right]+\left[U ; T_{1}^{\prime \prime}, T_{2}^{\prime \prime}\right]$, where $T_{1} \cup T_{2}, T_{1}^{\prime} \cup T_{2}^{\prime}$ and $T_{1}^{\prime \prime} \cup T_{2}^{\prime \prime}$ are as claspers $I, H$ and $X$ of Figure 2.8 .

Consider a term of type (1). By [4, Corollary 4.6], we have $\tilde{\lambda}_{n}\left(\left[U ; T_{1}, T_{2}\right]+\right.$ $\left.\left[U ; T_{1}^{\prime}, T_{2}^{\prime}\right]\right) \in \mathcal{M}_{2 n-1}$. The same holds for terms of type (2) by [4, Theorem 4.11].

This completes the proof.

By Theorem 1.1 and Proposition 6.4, we have a well-defined homomorphism

$$
\lambda_{n}: \operatorname{Br}\left(\bar{J}_{2 n}(n+1)\right) \rightarrow \overline{\mathcal{M}}_{2 n-2}
$$

by setting

$$
\lambda_{n}\left([L-U]_{J_{2 n+1}}\right):=\left[S^{3}-S_{(L,+1)}^{3}\right]_{\mathcal{M}_{2 n-1}}
$$

\subsection{Proof of Theorem 1.3}

First, we show that $\lambda_{n}$ actually takes its values in the connected part of the Ohtsuki filtration.

Recall from Section 5.2 that $\operatorname{Br}\left(\bar{J}_{2 n}(n+1)\right)$ is generated by elements $\left[U ; T_{\sigma} \cup \widetilde{T}_{\sigma^{\prime}}\right]$, for $\sigma, \sigma^{\prime} \in S_{n-1}$. Each component $U_{i}$ of $U$ intersects one disk-leaf $f_{i}$ of $T_{\sigma}$ and one disk-leaf $f_{i}^{\prime}$ of $T_{\sigma^{\prime}}$. Denote by $G_{\sigma, \sigma^{\prime}}$ the $Y_{2 n-2}$-graph obtained from $T_{\sigma} \cup \widetilde{T}_{\sigma^{\prime}}$ by connecting, for each $1 \leq i \leq n+1$, the edges incident to $f_{i}$ and $f_{i}^{\prime}$.

Algebraic $8 \mathcal{G}$ Geometric Topology, Volume 6 (2006) 
Lemma 6.5 For all $\sigma, \sigma^{\prime} \in S_{n-1}$,

$$
\lambda_{n}\left(\left[U ; T_{\sigma} \cup \widetilde{T}_{\sigma^{\prime}}\right]\right) \equiv\left[S^{3} ; G_{\sigma, \sigma^{\prime}}\right] \bmod \mathcal{M}_{2 n-1} .
$$

Consequently, we have

$$
\lambda_{n}\left(\operatorname{Br}\left(\bar{J}_{2 n}(n+1)\right)\right) \subset \operatorname{Co}\left(\overline{\mathcal{M}}_{2 n-2}\right) .
$$

Proof For any $\sigma, \sigma^{\prime} \in S_{n-1}$, we have

$$
\lambda_{n}\left(\left[U ; T_{\sigma} \cup \widetilde{T}_{\sigma^{\prime}}\right]\right)=-S_{G\left(T_{\sigma} \cup \widetilde{T}_{\sigma^{\prime}}\right)}^{3}+S_{G\left(T_{\sigma}\right)}^{3}+S_{G\left(T_{\sigma^{\prime}}\right)}^{3}-S^{3},
$$

where, if $F$ is a $C_{n}^{a}$-forest for $U, G(F)$ denotes the clasper obtained in $S^{3}$ by $(+1)$-framed surgery along $U$.

For all $\tau \in S_{n-1}, G\left(T_{\tau}\right)$ is a linear $Y_{n-1}$-tree whose leaves are all $(-1)$-special leaves. So by Theorem 3.2, there exists a union $G_{\tau}$ of $Y_{k}$-trees, $k \geq 2 n-2$ such that $S_{G\left(T_{\tau}\right)}^{3} \cong S_{G_{\tau}}^{3}$.

On the other hand, $G\left(T_{\sigma} \cup \widetilde{T}_{\sigma^{\prime}}\right)$ is obtained from $T_{\sigma} \cup \widetilde{T}_{\sigma^{\prime}}$ by replacing $f_{i} \cup f_{i}^{\prime}$ by a pair of Hopf-linked (-1)-framed leaves (as illustrated in Figure 6.2), for $1 \leq i \leq n+1$. By Habiro's move 7 and 2, $G\left(T_{\sigma} \cup \widetilde{T}_{\sigma^{\prime}}\right)$ is equivalent to the clasper $C$ obtained by

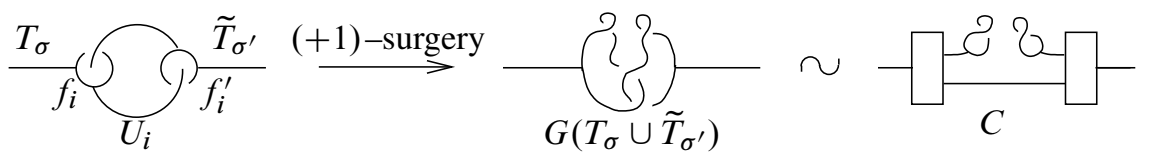

Figure 6.2: Performing $(+1)$-framed surgery along the unlink $U$

replacing each such pair of Hopf-linked leaves by two boxes as shown in Figure 6.2. By using the zip construction and Lemma 2.6, we obtain

$$
S_{C}^{3} \cong S_{G\left(T_{\sigma} \cup \tilde{T}_{\sigma^{\prime}}\right)}^{3} \sim_{Y_{2 n-1}} S_{G_{\sigma, \sigma^{\prime}} \cup G\left(T_{\sigma}\right) \cup G\left(\widetilde{T}_{\sigma^{\prime}}\right)}^{3} .
$$

It follows that

$$
\lambda_{n}\left(\left[U ; T_{\sigma} \cup \widetilde{T}_{\sigma^{\prime}}\right]\right) \equiv-S_{G_{\sigma, \sigma^{\prime}} \cup G_{\sigma} \cup G_{\sigma^{\prime}}}^{3}+S_{G_{\sigma}}^{3}+S_{G_{\sigma^{\prime}}}^{3}-S^{3} \bmod \mathcal{M}_{2 n-1} .
$$

By using the equality $S_{G_{\sigma, \sigma^{\prime}}}^{3} \cup G_{\sigma} \cup G_{\sigma^{\prime}}=\sum_{G^{\prime} \subseteq\left\{G_{\sigma, \sigma^{\prime}}, G_{\sigma}, G_{\sigma^{\prime}}\right\}}(-1)^{\left|G^{\prime}\right|}\left[S^{3} ; G^{\prime}\right]$, one can easily check that

$$
S_{G_{\sigma, \sigma^{\prime}} \cup G_{\sigma} \cup G_{\sigma^{\prime}}}^{3} \equiv S_{G_{\sigma, \sigma^{\prime}}}^{3}+S_{G_{\sigma}}^{3}+S_{G_{\sigma^{\prime}}}^{3}-2 S^{3} \bmod \mathcal{M}_{2 n-1} .
$$

(here we use the fact that $G_{\sigma, \sigma^{\prime}}$ and each connected component of $G_{\sigma}$ and $G_{\sigma^{\prime}}$ have degree $\geq 2 n-2)$. The result follows. 
Clearly, the composite $\alpha_{n-1} \lambda_{n}$ is the map

$$
\kappa_{n}: \operatorname{Br}\left(\bar{J}_{2 n}(n+1)\right) \longrightarrow \overline{\mathcal{S}}_{2 n-2}
$$

announced in the statement of Theorem 1.3. By Lemma 6.2, it suffices to show that $\lambda_{n}$ is an isomorphism to obtain the theorem. This is implied by the next lemma.

Lemma 6.6 For $n \geq 3$, the following diagram commutes:

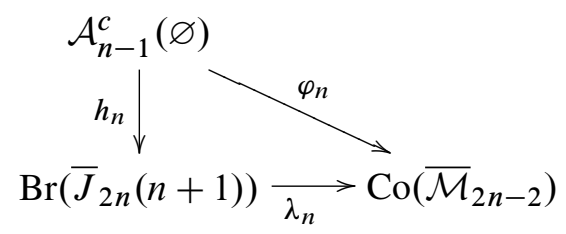

Proof As pointed out in [14, Section 3.5], one can easily check that $\mathcal{A}_{n-1}^{c}(\varnothing)$ is generated by the elements $\Gamma_{\sigma}$ depicted in Figure 6.3, for all $\sigma \in S_{n-1}$.

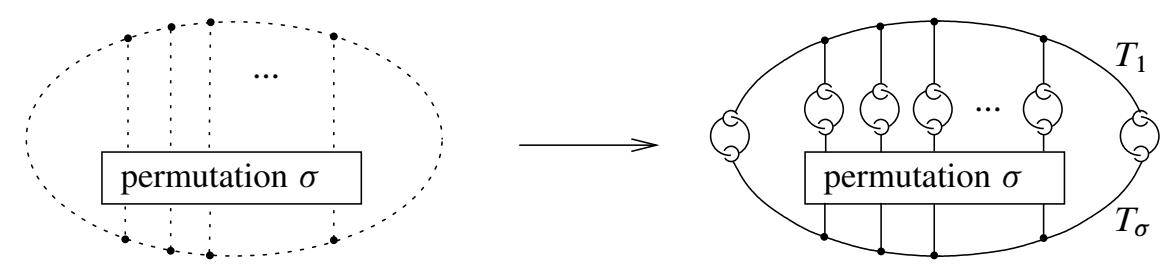

Figure 6.3: The connected trivalent diagram $\Gamma_{\sigma}$, and the two simple linear $C_{n}^{a}$-trees $T_{1}$ and $T_{\sigma}$

For such an element $\Gamma_{\sigma}$, a representative for $h_{n}\left(\Gamma_{\sigma}\right)$ is $\left[U ; T_{1} \cup T_{\sigma}\right]$, where $T_{1}$ and $T_{\sigma}$ are two $C_{n}^{a}$-trees for $U$ as represented in Figure 6.3. As seen in the proof of Lemma $6.5, \lambda_{n}\left(\left[U ; T_{1} \cup T_{\sigma}\right]\right)=\left[S^{3} ; G_{1, \sigma}\right]_{\mathcal{M}_{2 n-1}}$, where $G_{1, \sigma}$ is obtained by replacing each pair of disk-leaves intersecting the same component of $U$ by an edge. Clearly, this $Y_{2 n-2}$-graph satisfies $\varphi_{n}\left(\Gamma_{\sigma}\right)=\left[S^{3} ; G_{1, \sigma}\right]_{\mathcal{M}_{2 n-1}}$.

The various results proved of this section can be summed up in the following commutative diagram $(n \geq 2)$

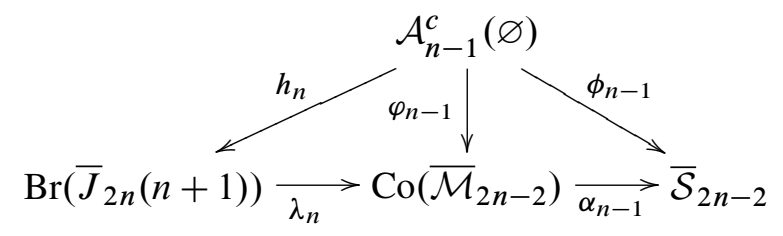

where all arrows are isomorphism over $\mathbf{Q}$.

Algebraic 8 Geometric Topology, Volume 6 (2006) 


\subsection{Brunnian links with vanishing Milnor invariants}

In this last subsection, we can work over the integers.

Habegger and Orr also studied finite type invariants of integral homology spheres obtained by $(+1)$-framed surgery along links in $S^{3}$. In particular, [10, Theorem 2.1] deals with $(+1)$-framed surgery along $l$-component Brunnian links with vanishing Milnor invariants of length $\leq 2 l-1$, and appears to have some similarities with our results.

Let $\operatorname{Br}^{l}\left(\bar{J}_{k}(n)\right)$ denote the $\mathbf{Z}$-submodule of $\bar{J}_{k}(n)$ generated by elements $[L-U]_{J_{k+1}}$ where $L$ is an $n$-component Brunnian link with vanishing Milnor invariants of length $\leq l$. Let $U_{(k)}$ denote the $k$-component unlink $U_{1} \cup \cdots \cup U_{k}$ in $S^{3}$. Let

$$
S_{n+1}: \operatorname{Br}\left(\bar{J}_{2 n}(n+1)\right) \longrightarrow \mathbf{Z} \mathcal{L}(n)
$$

be the map defined by

$$
S_{n+1}\left(\left[L-U_{(n+1)}\right]_{J_{2 n+1}}\right)=s_{n+1}(L)-U_{(n)},
$$

where $s_{n+1}(L)$ denotes the $n$-component link in $S^{3}$ obtained by $(+1)$-framed surgery along the $(n+1)^{t h}$ component of $L$. In particular, $s_{n+1}\left(U_{(n+1)}\right)=U_{(n)}$.

We can show that, for $n \geq 3$,

(1) $S_{n+1}\left(\operatorname{Br}\left(\bar{J}_{2 n}(n+1)\right)\right)=\operatorname{Br}^{2 n-1}\left(\bar{J}_{2 n-1}(n)\right)$

(2) $S_{n+1} \otimes \mathbf{Q}: \operatorname{Br}\left(\bar{J}_{2 n}(n+1)\right) \otimes \mathbf{Q} \rightarrow \operatorname{Br}^{2 n-1}\left(\bar{J}_{2 n-1}(n)\right) \otimes \mathbf{Q}$ is an isomorphism.

The proof involves the same technique as in the preceding section, and makes use of Theorem 6.1 of [9].

\section{The proof of Proposition 3.8}

In this section, we give the proof of Proposition 3.8. For that purpose, it is convenient to state a few more technical lemmas on claspers.

Lemma 7.1 The move of Figure 7.1 produces equivalent claspers.

This is an easy consequence of [12, Proposition 2.7].

Algebraic 83 Geometric Topology, Volume 6 (2006) 


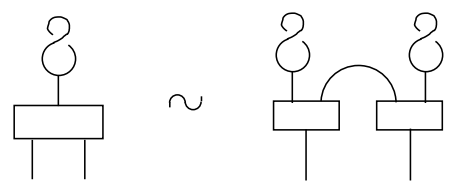

Figure 7.1

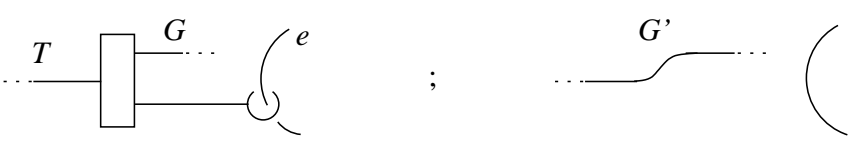

Figure 7.2

Lemma 7.2 Let $G$ be a clasper in a 3-manifold $M$ containing a $Y_{k}$-subtree $T$, $k \geq 1$, such that a branch of $T$ is incident to a box as shown in Figure 7.2. There, $e$ is an edge of $G$ which is not contained in $T$. Then

$$
M_{G} \sim_{Y_{k+1}} M_{G^{\prime}}
$$

where $G^{\prime}$ is the clasper depicted in the right-hand side of Figure 7.2.

The proof is omitted. It is straightforward, and uses Habiro's move 12 and a zip construction.

Lemma 7.3 Let $G$ be a clasper in a 3-manifold $M$ such that a 3-ball $B$ in $M$ intersects $G$ as depicted in Figure 7.3. There, the nodes $n_{1}$ and $n_{2}$ are both in a $Y_{k}$-subtree $T, k \geq 2$, and $e$ is an edge of $G$ which is not contained in $T$. Then

$$
M_{G} \sim_{Y_{k+1}} M_{G^{\prime}},
$$

where $G^{\prime}$ is identical to $G$ outside of $B$, where it is as shown in Figure 7.3.

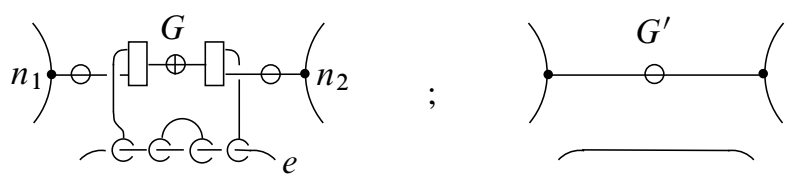

Figure 7.3

Algebraic 83 Geometric Topology, Volume 6 (2006) 
Proof By an isotopy, $G$ is seen to be equivalent to the clasper $G_{1}$ represented in Figure 7.4. By applying the move of [12, Figure 38] to $G_{1}$, and then applying Habiro's move 6 twice, we obtain the clasper $G_{2} \sim G_{1}$ of Figure 7.4. Consider the two I-shaped
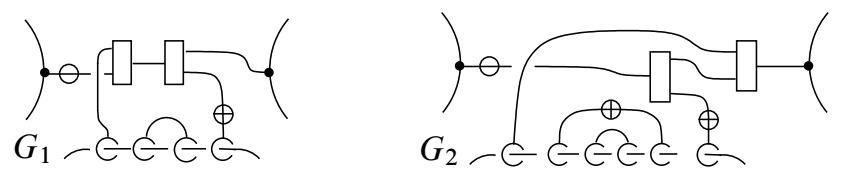

Figure 7.4

claspers $I_{1} \cup I_{2}$ of $G_{2}$ which appear in the figure. By Habiro's move 6 and 4, we have that $G_{2} \sim G_{2} \backslash\left(I_{1} \cup I_{2}\right)$. The result then follows from Lemma 7.2.

We can now prove Proposition 3.8.

Let $G$ be a linear $Y_{n}$-tree in a 3-manifold $M, n \geq 2$, with $n+2(-1)$-special leaves, and let $N$ denote an $s$-regular neighborhood $N$ of $G$. As noted previously, $N$ is a 3-ball in $M$.

By $(n-1)$ applications of Lemma 2.3, $G$ is equivalent to the clasper $\widetilde{G}$ represented in Figure 7.5. The first step of this proof is to show the following.

Claim 7.4 We have

$$
\widetilde{G} \sim C,
$$

in $N$, where $C$ is the clasper containing a $Y_{2 n}$-subtree represented in Figure 7.5.

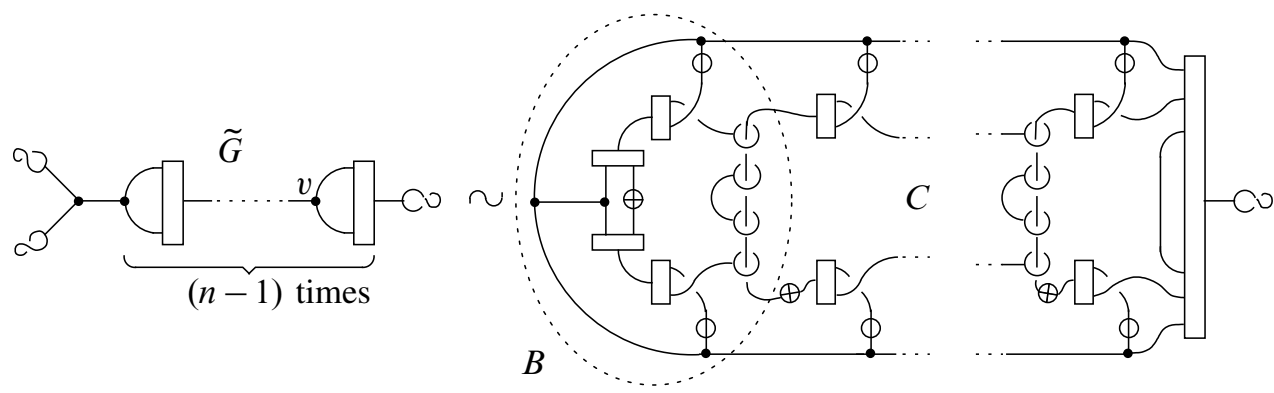

Figure 7.5 
Proof Consider the box of $\widetilde{G}$ which is connected to one (-1)-special leaf. This box is connected to a node $v$ by two edges. By applying Lemma 2.4 at $v$, and Lemma 2.5, we obtain the clasper represented in Figure 7.6 (a). Then apply recursively Lemma 2.4 and Habiro's move 6, as shown in Figure 7.6 (b), until we obtain a clasper $G^{\prime} \sim \tilde{G}$ with only one node connected to two (-1)-special leaves. See in Figure 7.6 (c). By

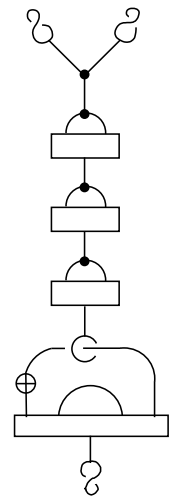

(a)

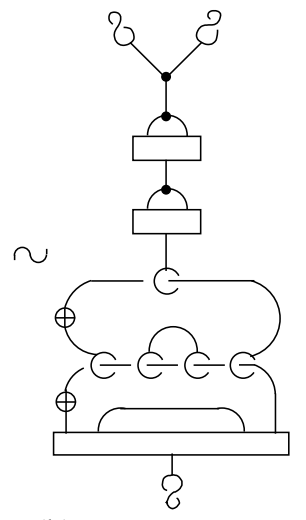

(b)

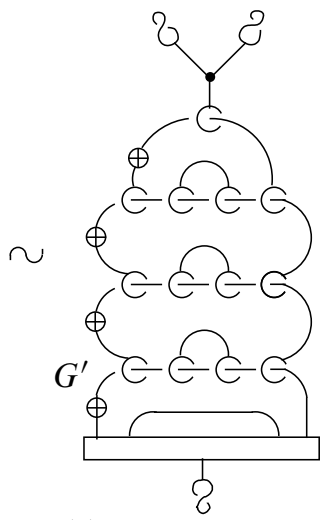

(c)

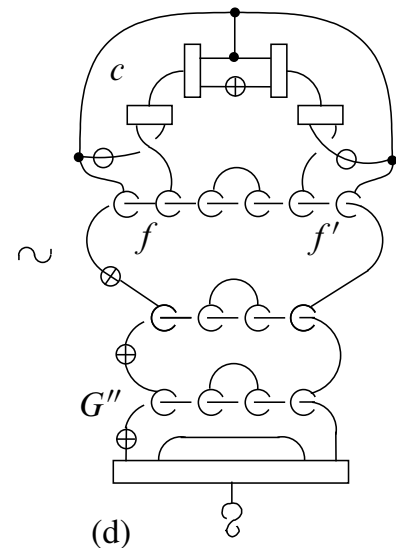

(d)

Figure 7.6: Here, for simplicity, we consider the case $n=5$.

applying the move of Figure 3.1 and Habiro's move 6, we have $G^{\prime} \sim G^{\prime \prime}$, where $G^{\prime \prime}$ contains a component $c$ with 4 nodes and with two leaves $f$ and $f^{\prime}$ lacing an edge $e$ - see Figure 7.6 (d). ${ }^{7}$ We can apply Habiro's move 12 to these two leaves, and then Habiro's move 6 to create two new leaves lacing an edge. Apply recursively these two moves until no new leaf lacing an edge is created: the result is the desired clasper $C$ which contains a $Y_{2 n}$-subtree $T$, as represented in Figure 7.5.

Consider in $N$ a 3-ball $B$ which intersects $C$ as depicted. By several applications of the move of [12, Figure 38] and of Habiro's move 6, we obtain the clasper $G_{1} \sim G$ which is identical to $C$ outside $B$, where it is as shown in Figure 7.7. By Habiro's move 6 and 4, we can freely remove the pair of I-shaped claspers which appear in the figure (see the proof of Lemma 7.3). By further applying four times Lemma 7.2, we thus obtain the clasper $G_{2}$ of Figure 7.7, which satisfies $N_{G_{2}} \sim_{Y_{2 n+1}} N_{G_{1}}$. By an

\footnotetext{
${ }^{7}$ Here we say that a leaf of a clasper $G$ laces an edge if it forms an unknot which bounds a disk $D$ with respect to which it is 0 -framed, such that the interior of $D$ intersects $G$ once, transversally, at an edge.
}

Algebraic 8 Geometric Topology, Volume 6 (2006) 

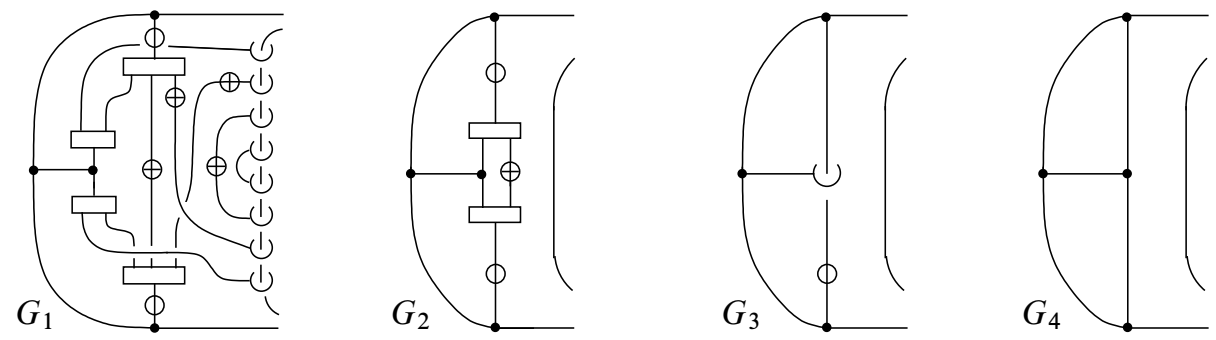

Figure 7.7: These four claspers are identical to $C$ outside $B$.

isotopy, we can apply Habiro's move 12 to show that $N_{G_{2}} \sim N_{G_{3}}$, where $G_{3}$ is as shown in Figure 7.7. By using [20, page 398], we obtain $N_{G_{3}} \sim_{Y_{2 n+1}} N_{G_{4}} \cdot{ }^{8}$

Observe that $G_{4}$ satisfies the hypothesis of Lemma 7.3. Actually, we can apply Lemma 7.3 recursively $(n-3)$ times. By further applying, to the resulting clasper, strictly the same arguments as in the proof of Lemma 7.3, we obtain $N_{G_{4}} \sim_{Y_{2 n+1}} N_{G_{5}}$, where $G_{5}$ is the clasper shown in Figure 7.8. It follows, by the zip construction and Lemma 2.6, that

$$
N_{G_{5}} \sim_{Y_{2 n+1}} N_{G_{6} \cup G_{7}},
$$

where $G_{6}$ and $G_{7}$ are two disjoint claspers in $N$ as represented in Figure 7.8.
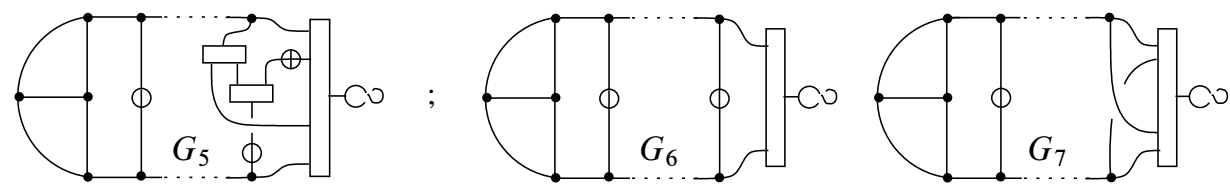

Figure 7.8

By Lemma 7.1 and Theorem 3.2 (for $l=1$ ), it is not hard to check that $N_{G_{7}} \sim_{Y_{2 n+1}} N$ and that $N_{G_{6}} \sim_{Y_{2 n+1}} N_{\Theta_{n}}$.

This concludes the proof of Proposition 3.8.

\section{References}

[1] D Bar-Natan, On the Vassiliev knot invariants, Topology 34 (1995) 423-472 MR1318886

\footnotetext{
${ }^{8}$ We use the up-most figure of [20, page 398]. The arguments given there are for graph claspers, but they can be used in our situation.
}

Algebraic 8 Geometric Topology, Volume 6 (2006) 
[2] T D Cochran, P Melvin, Finite type invariants of 3-manifolds, Invent. Math. 140 (2000) 45-100 MR1779798

[3] J Conant, P Teichner, Grope cobordism of classical knots, Topology 43 (2004) 119156 MR2030589

[4] S Garoufalidis, M Goussarov, M Polyak, Calculus of clovers and finite type invariants of 3-manifolds, Geom. Topol. 5 (2001) 75-108 MR1812435

[5] S Garoufalidis, J Levine, Finite type 3-manifold invariants, the mapping class group and blinks, J. Differential Geom. 47 (1997) 257-320 MR1601612

[6] S Garoufalidis, T Ohtsuki, On finite type 3-manifold invariants. III. Manifold weight systems, Topology 37 (1998) 227-243 MR1489202

[7] M Goussarov, Finite type invariants and $n$-equivalence of 3-manifolds, C. R. Acad. Sci. Paris Sér. I Math. 329 (1999) 517-522 MR1715131

[8] M Goussarov, Variations of knotted graphs. The geometric technique of n-equivalence, Algebra i Analiz 12 (2000) 79-125 MR1793618 (Russian) translation in St. Petersburg Math. J. 12 (2001) no. 4, 569-604

[9] N Habegger, G Masbaum, The Kontsevich integral and Milnor's invariants, Topology 39 (2000) 1253-1289 MR1783857

[10] N Habegger, K E Orr, Finite type three manifold invariants-realization and vanishing, J. Knot Theory Ramifications 8 (1999) 1001-1007 MR1723435

[11] K Habiro, Brunnian links, claspers, and Goussarov-Vassiliev finite type invariants, to appear in Math. Proc. Camb. Phil. Soc.

[12] K Habiro, Claspers and finite type invariants of links, Geom. Topol. 4 (2000) 1-83 MR1735632

[13] K Habiro, J-B Meilhan, Finite type invariants and Milnor invariants for Brunnian links arXiv:math.GT/0510534

[14] K Habiro, J-B Meilhan, On the Kontsevich integral of Brunnian links, Algebr. Geom. Topol. 6 (2006) 1399-1412 MR2253452

[15] T T Q Le, An invariant of integral homology 3-spheres which is universal for all finite type invariants, from: "Solitons, geometry, and topology: on the crossroad", (V Buchstaber, S Novikov, editors), Amer. Math. Soc. Transl. Ser. 2 179, Amer. Math. Soc., Providence, RI (1997) 75-100 MR1437158

[16] S V Matveev, Generalized surgeries of three-dimensional manifolds and representations of homology spheres, Mat. Zametki 42 (1987) 268-278, 345 MR915115

[17] J-B Meilhan, Invariants de type fini des cylindres d'homologie et des string links, $\mathrm{PhD}$ thesis, Université de Nantes (2003)

[18] H A Miyazawa, A Yasuhara, Classification of $n$-component Brunnian links up to $C_{n}$-move, Topology Appl. 153 (2006) 1643-1650 MR2227018 
[19] T Ohtsuki, Finite type invariants of integral homology 3-spheres, J. Knot Theory Ramifications 5 (1996) 101-115 MR1373813

[20] T Ohtsuki, Quantum invariants, Series on Knots and Everything 29, World Scientific Publishing Co., River Edge, NJ (2002) MR1881401A study of knots, 3-manifolds, and their sets

Research Institute for Mathematical Sciences, Kyoto University

Kyoto 606-8502, Japan

meilhan@kurims.kyoto-u.ac.jp

Received: 30 May 2006 